\title{
Small Mass Nanopteron Traveling Waves in Mass-in-Mass Lattices with Cubic FPUT Potential
}

\author{
Timothy E. Faver ${ }^{1}$ (DD
}

Received: 19 November 2019 / Revised: 5 June 2020 / Published online: 7 July 2020

(c) The Author(s) 2020

\begin{abstract}
The mass-in-mass (MiM) lattice consists of an infinite chain of identical beads that are both nonlinearly coupled to their nearest neighbors and linearly coupled to a distinct resonator particle; it serves as a prototypical model of wave propagation in granular crystals and metamaterials. We study traveling waves in an MiM lattice whose bead interaction is governed by the cubic Fermi-Pasta-Ulam-Tsingou potential and whose resonator mass is small compared to the bead mass. Excluding a countable number of "antiresonance" resonator masses accumulating at 0 , we prove the existence of nanopteron traveling waves in this small mass limit. The profiles of these waves consist of the superposition of an exponentially localized core and a small amplitude periodic oscillation that itself is a traveling wave profile for the lattice. Our arguments use functional analytic techniques originally developed by Beale for a capillary-gravity water wave problem and recently employed in a number of related nanopteron constructions in diatomic Fermi-Pasta-Ulam-Tsingou lattices.
\end{abstract}

Keywords FPU · FPUT · Granular chain · Granular metamaterial · Mass-in-mass lattice · Mass-with-mass lattice $\cdot$ Nanopteron $\cdot$ Solitary wave

Mathematics Subject Classification 35C07 $\cdot 37 \mathrm{~K} 60 \cdot 35 \mathrm{~B} 20 \cdot 35 \mathrm{~B} 40$

\section{Introduction}

\subsection{The Mass-in-Mass Lattice}

Consider an infinite chain of particles, which we call "beads," each normalized to have mass 1 , arranged on a horizontal line and connected by identical springs whose potential is $\mathscr{V} \in \mathscr{C}^{1}(\mathbb{R})$. Linearly coupled to each bead is a "resonator" particle of mass $\mu>0$. The

I acknowledge support from the Netherlands Organization for Scientific Research (NWO) (Grant 639.032.612).

$凶$ Timothy E. Faver

t.e.faver@math.leidenuniv.nl

1 Mathematical Institute, University of Leiden, P.O. Box 9512, 2300 RA Leiden, The Netherlands 


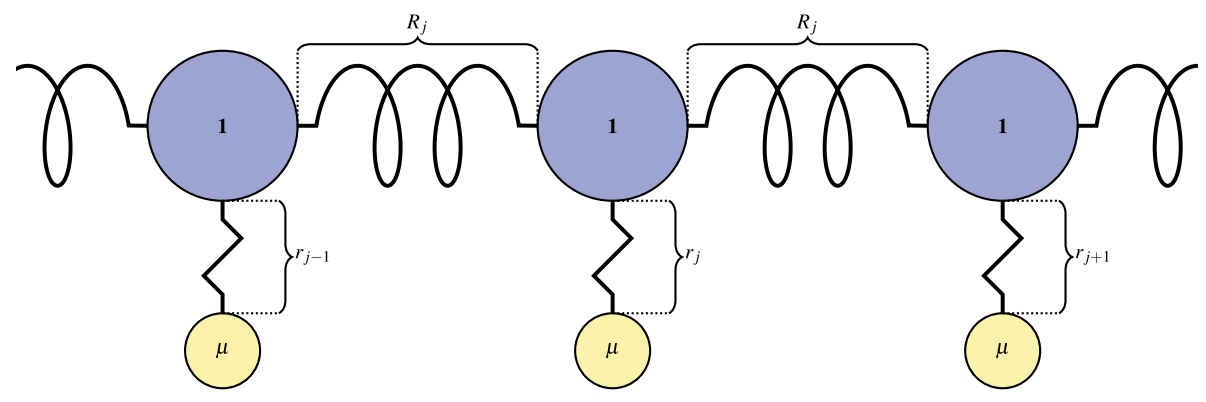

Fig. 1 The MiM lattice

springs connecting the beads to the resonators are all identical and exert the force $\kappa r$ when stretched a distance $r$. This construct is called a mass-in-mass (MiM) ${ }^{1}$ lattice.

Denote by $U_{j}$ the position of the center of mass of the $j$ th bead and by $u_{j}$ the position of the center of mass of the $j$ th resonator, with $j \in \mathbb{Z}$. Then Newton's law implies that

$$
\left\{\begin{array}{l}
\ddot{U}_{j}=\mathscr{V}^{\prime}\left(U_{j+1}-U_{j}\right)-\mathscr{V}^{\prime}\left(U_{j}-U_{j-1}\right)+\kappa\left(u_{j}-U_{j}\right) \\
\mu \ddot{u}_{j}=\kappa\left(U_{j}-u_{j}\right) .
\end{array}\right.
$$

We will rewrite (1.1) in terms of relative displacements. Set

$$
R_{j}:=U_{j+1}-U_{j} \quad \text { and } \quad r_{j}:=U_{j}-u_{j} .
$$

That is, $R_{j}$ is the distance between the $j$ th and the $(j+1)$ st bead and $r_{j}$ is the distance between the $j$ th bead and its attached resonator. Then

$$
\left\{\begin{array}{l}
\ddot{R}_{j}=\mathscr{V}^{\prime}\left(R_{j+1}\right)-2 \mathscr{V}^{\prime}\left(R_{j}\right)+\mathscr{V}^{\prime}\left(R_{j-1}\right)-\kappa\left(r_{j+1}-r_{j}\right) \\
\mu \ddot{r}_{j}=\mu\left[\mathscr{V}^{\prime}\left(R_{j}\right)-\mathscr{V}^{\prime}\left(R_{j-1}\right)\right]-\kappa(1+\mu) r_{j} .
\end{array}\right.
$$

See Fig. 1 for a sketch of this set-up. We are interested in traveling waves, and so we make the ansatz

$$
r_{j}(t)= \begin{cases}p_{1}(j-c t), & j \text { is odd } \\ p_{2}(j-c t), & j \text { is even, }\end{cases}
$$

where the profiles $p_{1}$ and $p_{2}$ are functions of a real variable and $c \in \mathbb{R}$ is the wave speed.

We will ultimately choose $\mathscr{V}$ to be the "cubic" potential

$$
\mathscr{V}(r)=\mathscr{V}_{\text {cubic }}(r):=\frac{r^{2}}{2}+\frac{r^{3}}{3} .
$$

We discuss the reasons for this election, as well as a survey of other relevant potentials, in Sect. 1.2.

An MiM lattice is an example of a granular crystal, or granular chain, which is a lattice of closely packed particles whose behavior chiefly depends on the interactions between

\footnotetext{
1 Some applications specify that the resonator is coupled to the bead internally, in which case the resonator is genuinely "in" the bead; others maintain the resonator outside the bead and call their lattice a "mass-with-mass" lattice.
} 
neighboring particles and the contact forces that they exert on each other. In a prototypical experiment, a finite, one-dimensional chain of particles is constructed; the chain is then struck at one end by another particle, and the resulting motion of the chain is measured [6]. Common to these experiments is an interest in the propagation of traveling waves through the granular crystal, which are waves whose form is given by a steadily translating profile [38,39].

The appeal of the MiM lattice in modeling arises its material tunability; one can vary the spring forces from forces with a dominant linear term (the "precompression" case) to highly nonlinear ones (no precompression), as well as the mass values, incorporating periodic structures and models with material defects. For example, the articles [4] and [20] describe numerical and physical experiments on a finite MiM lattice consisting of a single hollow aluminum bead with an internal solid steel resonator connected to the shell by a plastic structure; polycarbonate rods connect the bead to guide plates and permit it to move. The resonator acts to absorb vibrations and reinforces the structural integrity of composite materials. The MiM lattice need not be precisely a chain of particles and springs; one incarnation is the "woodpile" lattice of [32], where the lattice "beads" are rods made of fused quartz, and the lattice itself assembled by stacking the rods in an orthogonal configuration. Here, the internal vibrations in the rods serve as the internal resonator. As representative of the diversity of applications of MiM lattices, we mention that they arise in constructing sensors for bone elasticity [52] and ultrasonic scans [42], determining of the setting time for cement [40], and modeling of switches and logic gates [33].

\subsection{Reduction to Monatomic Lattices}

It is clear that if we take $\mu=0$, then the small resonator in the MiM lattice vanishes, and we are left with a monatomic Fermi-Pasta-Ulam-Tsingou [8,15] lattice with spring potential $\mathscr{V}$. Depending on the spring potential selected and the wave speed regime in which one works, the monatomic FPUT lattice is known to possess a variety of solitary traveling waves, i.e., traveling waves whose profiles vanish at spatial infinity. See Pankov [41] for a broad overview of traveling waves in monatomic and polyatomic lattices, including, but not limited to, solitary waves, and Kevrekidis [30] for an overview of their applications (both overlapping with and distinct from those for MiM lattices discussed previously).

Our intention is to perturb from a monatomic solitary wave, in an appropriate sense, and construct traveling wave solutions for the MiM lattice when $\mu$ is small. We make this precise in Sect. 2. Solitary traveling waves for the monatomic FPUT lattice have profiles $\rho$ such that putting

$$
R_{j}(t)=\rho(j-c t)
$$

solves

$$
\ddot{R}_{j}=\mathscr{V}^{\prime}\left(R_{j+1}\right)-2 \mathscr{V}^{\prime}\left(R_{j}\right)+\mathscr{V}^{\prime}\left(R_{j-1}\right)
$$

Equivalently, $\rho$ must satisfy

$$
c^{2} \rho^{\prime \prime}-A \mathscr{V}^{\prime}(\rho)=0, \quad(A \rho)(x):=\rho(x+1)-2 \rho(x)+\rho(x-1) .
$$

We highlight four families of bead potentials $\mathscr{V}$ and wave speeds $c$ for which the monatomic FPUT lattice with spring potential $\mathscr{V}$ is known to have solitary traveling wave solutions.

(i) Friesecke and Pego [16] show that (1.5) has an exponentially localized solution for $\mathscr{V} \in \mathscr{C}^{4}(\mathbb{R})$ with $\mathscr{V}(0)=\mathscr{V}^{\prime}(0)=0, \mathscr{V}^{\prime \prime}(0)>0$, and $\mathscr{V}^{\prime \prime \prime}(0) \neq 0$, with $c$ chosen in 
the "near-sonic" regime $|c| \gtrsim \sqrt{V^{\prime \prime}(0)}$. The cubic potential $\mathscr{V}_{\text {cubic }}$ from (1.4), which was one of the original potentials studied by Fermi, Pasta, Ulam, and Tsingou in [15], certainly satisfies these conditions, as do the Toda [47] and Lennard-Jones potentials. The Friesecke-Pego solution turns out to be a small perturbation in $H^{1}$ of a $\mathrm{KdV}$ sech $^{2}$-type solitary wave.

(ii) Friesecke and Wattis [17] take $\mathscr{V} \in \mathscr{C}^{2}(\mathbb{R})$ to be "superquadratic" with $r \mapsto \mathscr{V}(r) / r^{2}$ strictly increasing on either $(-\infty, 0)$ or $(0, \infty)$. The wave speed $c$ is supersonic in that $|c|>\sqrt{\mathscr{V}^{\prime \prime}(0)}$ but otherwise not specified. Their methods apply to a plethora of potentials, including the cubic FPUT.

(iii) Herrmann and Matthies [22-24] construct localized solutions in the "high-energy" limit, i.e., with $|c|$ large, for a class of singular convex potentials that resemble the Lennard-Jones potential.

(iv) The bead potential most often associated with MiM lattices in the literature is the Hertzian potential, which we now describe in some detail. For $r \in \mathbb{R}$, set

$$
[r]_{+}:=\left\{\begin{array}{l}
r, r \geq 0 \\
0, r<0,
\end{array}\right.
$$

and, for $\epsilon_{0} \geq 0$ and $p>1$, take $\mathscr{Y}$ Hertz to be the potential satisfying

$$
\mathscr{V}_{\text {Hertz }}^{\prime}(r):=\left[\epsilon_{0}+r\right]_{+}^{p} \text {. }
$$

When $\epsilon_{0}>0$, the potential models lattices experiencing a "precompression" force in which there is an additional force pressing on the ends of the lattice [39]. MacKay [37], Ji and Hong [28], English and Pego [9], and Kevrekidis and Stefanov [44,45] all have established the existence of solitary wave in Hertzian monatomic lattices for different values of $p$ and $\epsilon_{0}$.

Moreover, Kevrekidis, Stefanov, and Xu show that for certain "antiresonance" values of the resonator mass $\mu$, the MiM lattice with Hertzian bead potential (i.e., $\mathscr{V}=\mathscr{V} /$ Hertz in (1.1)) retains solitary traveling waves. In a forthcoming paper, Faver, Goodman, and Wright [12] do the same for MiM lattices with a potential similar to the Friesecke-Pego class of (i). We discuss these MiM solitary waves in much greater detail in Sect. 4.3.

We have chosen the cubic FPUT potential $\mathscr{V}_{\text {cubic }}$ from (1.4) to govern the interaction of the beads in the MiM lattice studied in this paper for two reasons. First, the simple "linear + quadratic" structure ${ }^{2}$ that the spring forces consequently possess puts us in concert with a host of previous papers studying traveling waves in lattices, namely $[11,13,14,16,25]$. Second, we view the cubic FPUT potential as an approximation to the Hertzian potential with precompression. Taylor-expanding $\mathscr{V}_{\text {Hertz }}^{\prime}$ from (1.6) to second order, one has

$$
\mathscr{V}_{\text {Hertz }}^{\prime}(r) \approx \epsilon_{0}^{p}+a r+b r^{2}
$$

for constants $a$ and $b$, and clearly the constant term $\epsilon_{0}^{p}$ is annihilated by the differences in the first equation in both (1.1) and (1.2). With the bead potential now fixed, we next discuss the selection of the precise monatomic solution from which we will perturb when $\mu$ is small.

\footnotetext{
${ }^{2}$ We expect could add higher-order terms to $\mathscr{V}_{\text {cubic }}$ and use the composition operator techniques from [11] to solve the technically more complicated problem that then arises. For simplicity, however, we omit these terms here. An interesting generalization for future study would be to add nonlinear terms to the bead-resonator force; see Sect. 4.7.
} 


\subsection{The Monatomic Profile}

Rather than restrict ourselves to a Friesecke-Pego [16] or Friesecke-Wattis [17] profile, both of which exist for the cubic potential $\mathscr{V}_{\text {cubic }}$, we instead hypothesize the existence of a monatomic solution with some general properties. First, we need to specify the function spaces in which we work.

For $q>0$, let

$$
H_{q}^{r}=\left\{f \in H^{r} \mid \cosh ^{q}(\cdot) f \in H^{r}\right\}, \quad\|f\|_{r, q}=\|f\|_{H_{q}^{r}}:=\left\|\cosh ^{q}(\cdot) f\right\|_{H^{r}}
$$

and set

$$
\begin{array}{r}
E_{q}^{r}:=H_{q}^{r} \cap\{\text { even functions }\}, \\
O_{q}^{r}:=H_{q}^{r} \cap\{\text { odd functions }\},
\end{array}
$$

and

$$
E_{q, 0}^{r}:=\left\{f \in E_{q}^{r} \mid \widehat{f}(0)=0\right\},
$$

where $\widehat{f}$ is the Fourier transform of $f$ (see Appendix A.1 for our notations and conventions for Fourier analysis). These exponentially localized spaces are all Banach spaces under the norm $\|\cdot\|_{r, q}$; see Appendix C of [10] for some elaborations on their properties.

Hypothesis 1 There exist a real number $c$ with $|c|>1$ such that the following properties hold.

(i) There exist $q_{c}>0$ and a positive solution $\sigma_{c} \in E_{q_{c}}^{2}$ to the monatomic FPUT traveling wave problem (1.5) with $\mathscr{V}=\mathscr{V}_{\text {cubic. }}$

(ii) The linearization of the monatomic traveling wave problem (1.5) at $\sigma_{c}$, which is the operator

$$
\mathscr{H}_{c} f:=c^{2} f^{\prime \prime}-A\left(1+2 \sigma_{c}\right) f
$$

is invertible from $E_{q_{c}}^{2} \rightarrow E_{q_{c}, 0}^{0}$.

When $|c| \gtrsim 1$ and $\sigma_{c}$ is the Friesecke-Pego [16] solitary wave, Hoffman and Wright have shown the invertibility of $\mathscr{H}_{c}$ in Proposition 3.1 of [25]. Herrmann and Matthies discuss the invertibility of the analogous operator for their singular potential in the high-energy limit in [23]. So, we feel there is solid precedent for requiring the invertibility of $\mathscr{H}_{c}$ in our hypothesis. The condition that $|c|>1$ will be important at a technical level deep in the appendices (see the proof of Proposition 5, for example), but otherwise it plays no role; we hope that making the existence of a monatomic FPUT solution a hypothesis will put us in further dialogue with existing and future monatomic results, instead of just restricting, say, to the near-sonic case $|c| \gg 1$.

A straightforward bootstrapping argument shows that $\sigma_{c} \in \cap_{r=0}^{\infty} E_{q_{c}}^{r}$. Less trivial is the following extension of the invertibility of $\mathscr{H}_{c}$ over a range of weights. This is stated and proved as Proposition 5.1 in [13].

Proposition 1 There exist $q_{1}(c)<q_{2}(c) \leq q_{c}$ such that for $q \in\left[q_{1}(c), q_{2}(c)\right]$ and $r \geq 0$, the operator $\mathscr{H}_{c}$ is invertible from $E_{q}^{r+2}$ to $E_{q}^{r}$. 


\subsection{Nanopterons and the Main Result}

Our instinctive intention is to look for traveling wave profiles $\left(p_{1}, p_{2}\right)$ for the MiM lattice with cubic potential that are small, exponentially localized perturbations of $\left(\sigma_{c}, 0\right)$. However, experience with traveling waves in heterogeneous FPUT lattices, in which the FPUT lattice no longer has identical springs and masses but either alternating masses (the mass dimer or diatomic lattice) or alternating spring potentials (the spring dimer), suggests that material heterogeneity in lattices induces the classical solitary wave to perturb into a nonlocal solitary wave. Specifically, we refer to the long wave limit studied by Faver and Wright [14] for the mass dimer and again by Faver [11] for the spring dimer, the small mass limit in mass dimers of Hoffman and Wright [25], and the equal mass limit of Faver and Hupkes [13], again for the mass dimer. We also note a variety of numerical studies of nanopeterons in mass and spring dimer lattices, including [19,21,35,36,43,48].

The traveling waves for these dimers under the relevant physical limits do not vanish at infinity but instead asymptote to small amplitude periodic "ripples," which themselves are exact traveling waves, too. In the long wave and small mass problems, a singular perturbation in the traveling wave equations causes the amplitude of the ripple to be small beyond all orders of the relevant small parameter, and thus these nonlocal solitary waves are nanopterons, in Boyd's terminology [5]; in the equal mass limit there is no singular perturbation, and the ripples are only, ostensibly, algebraically small.

Furthermore, there is a considerable body of numerical evidence specifically for MiM lattices indicating the existence of nanopterons therein; see the numerical experiments and results discussed in [18,31,32,50,51]. The article [18], for example, suggests that the resonator can act as a "defect" to trap some of an MiM traveling wave's energy in localized oscillations.

Motivated, then, by this numerical evidence-in particular the observations that Kevrekidis, Stefanov, and $\mathrm{Xu}$ [31] made of nanopteron behavior for resonator mass values $\mu$ away from the antiresonance cases - and the success of Hoffman and Wright in studying the diatomic FPUT small mass problem, we will construct nanopteron traveling waves for the MiM lattice whose bead interactions have the cubic FPUT potential. Here, then, is our main theorem, proved precisely as Theorem 3 . We sketch the nanopteron profile $p_{1}$ from this theorem in Fig. 2.

Theorem 1 Suppose that $|c|>1$ satisfies Hypothesis 1 and $\kappa>0$. Then there exist $\mu_{\star}>0$ and an open set $\mathrm{M}_{c, \kappa} \subseteq\left(0, \mu_{\star}\right)$ with the following properties.

(i) $0 \in \overline{\mathbf{M}_{c, \kappa}}$.

(ii) If $\mu \in \mathrm{M}_{c, \kappa}$, then there are functions $\varphi_{1}^{\mu}, \varphi_{2}^{\mu}, \Upsilon_{1}^{\mu}, \Upsilon_{2}^{\mu} \in \mathscr{C}^{\infty}(\mathbb{R})$ such that putting

$p_{1}:=\sigma_{c}\left(x+\frac{1}{2}\right)+\varphi_{1}^{\mu}\left(x+\frac{1}{2}\right)+\Upsilon_{1}^{\mu}\left(x+\frac{1}{2}\right) \quad$ and $\quad p_{2}:=\varphi_{2}^{\mu}(x)+\Upsilon_{2}^{\mu}(x)$

gives traveling wave profiles for the MiM equations (1.2) with cubic FPUT potential (1.4).

(iii) The threshold $\mu_{\star}$ above is small enough so that the following asymptotic estimates hold. The functions $\varphi_{1}^{\mu}$ and $\varphi_{2}^{\mu}$ are periodic with frequency $\mathscr{O}\left(\mu^{-1 / 2}\right)$ and amplitude small beyond all orders of $\mu$; the functions $\Upsilon_{1}^{\mu}$ and $\Upsilon_{2}^{\mu}$ are exponentially localized with amplitude $\mathscr{O}(\mu)$.

The additional shift by $1 / 2$ in $p_{1}$ in (1.8) is a consequence of a symmetrization choice that we make in our traveling wave problem in (2.2). The restriction of $\mu$ to the set $\mathbf{M}_{c, \kappa}$ is 


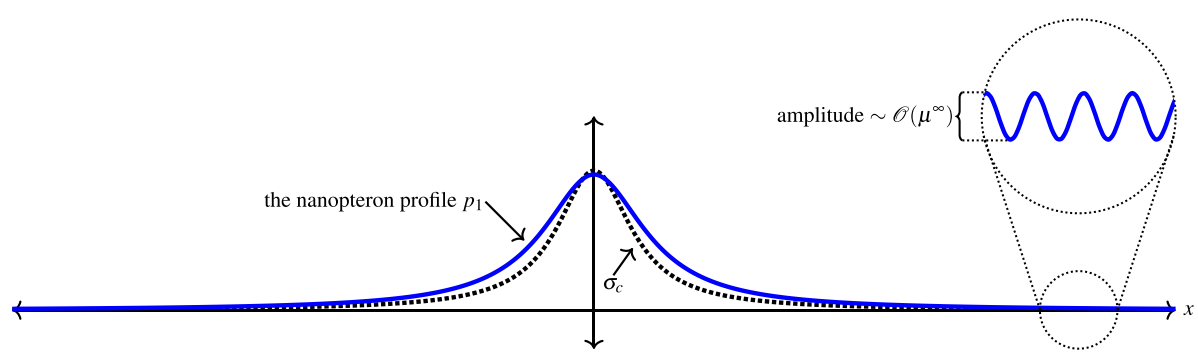

Fig. 2 The nanopteron traveling wave profile $p_{1}$. By $\mathscr{O}\left(\mu^{\infty}\right)$ we mean "small beyond all orders of $\mu$ "

nontrivial and technical and is discussed at length in Sects. 4.2 and 4.3; the precise definition of $\mathbf{M}_{c, \kappa}$ appears in Lemma 6 . Roughly, $\mathbf{M}_{c, \kappa}$ excludes from the interval $(0,1)$ the antiresonance values of $\mu$ at which [31] and [12] find solitary waves.

\subsection{Remarks on Notation}

We outline some conventions that will be frequently used in the following arguments.

- We regard $c$ and $\kappa$ as fixed throughout this problem. All constants and estimates are allowed to depend on both $c$ and $\kappa$. We occasionally indicate dependence of an operator on $c$ to highlight the relationship to wave speed.

- All constants are independent of $\mu$, and if a constant does depend on some other parameter (which is not $c$ or $\kappa$ ), we indicate that with function notation, e.g., if the constant $C$ depends on the parameters $q$ and $r$, then we write $C=C(q, r)$.

- If $x_{\mu}$ is a real number that depends on $\mu$, and if $p \in \mathbb{R}$, then we write $x=\mathscr{O}\left(\mu^{r}\right)$ if there are constants $\mu_{r}, C(r)>0$ such that $\left|x_{\mu}\right| \leq C(r)|\mu|^{r}$ for $|\mu| \leq \mu_{r}$. We write $x_{\mu}=\mathscr{O}\left(\mu^{\infty}\right)$ if there are constants $\mu_{\infty}, C(r)>0$ such that $\left|x_{\mu}\right| \leq C(r)|\mu|^{r}$ for each $r \geq 0$ and $|\mu| \leq \mu_{\infty}$.

- If $\mathscr{X}$ and $\mathscr{Y}$ are normed spaces, we write $\mathbf{B}(\mathscr{X}, \mathscr{Y})$ for the space of bounded linear operators from $\mathscr{X}$ to $\mathscr{Y}$, and we define $\mathbf{B}(\mathscr{X}):=\mathbf{B}(\mathscr{X}, \mathscr{X})$.

\section{The Traveling Wave Problem}

\subsection{The Traveling Wave Equations}

With $\mathbf{p}=\left(p_{1}, p_{2}\right)$ and $I_{\mu}:=\operatorname{diag}(1, \mu)$, we use the traveling wave ansatz (1.3) to convert our relative displacement equations (1.2) to

$$
c^{2} I_{\mu} \mathbf{p}^{\prime \prime}+\left[\begin{array}{cc}
-\left(S^{1}-2+S^{-1}\right) & \kappa\left(S^{1}-1\right) \\
\mu\left(S^{-1}-1\right) & \kappa(1+\mu)
\end{array}\right] \mathbf{p}+\left(\begin{array}{c}
-\left(S^{1}-2+S^{-1}\right) p_{1}^{2} \\
\mu\left(S^{-1}-1\right) p_{1}^{2}
\end{array}\right)=0 .
$$

Motivated by the traveling wave ansatz in [22], we can "symmetrize" this equation by changing variables to

$$
\rho:=\left[\begin{array}{cr}
S^{-1 / 2} & 0 \\
0 & 1
\end{array}\right] \mathbf{p}
$$

The extra shift by $1 / 2$ in our final form of the profiles in (1.8) is an artifact of this extra change of variables. 
Then (2.1) is equivalent to

$$
\underbrace{c^{2} I_{\mu} \rho^{\prime \prime}+\mathscr{D}_{\mu} \rho+\mathscr{D}_{\mu} \mathscr{Q}(\boldsymbol{\rho}, \boldsymbol{\rho})}_{\mathscr{G}_{c}(\boldsymbol{\rho}, \mu)}=0,
$$

where

$$
\mathscr{D}_{\mu}:=\left[\begin{array}{cc}
-A & \kappa \delta \\
-\mu \delta & \kappa(1+\mu)
\end{array}\right], \quad A:=S^{1}-2+S^{-1}, \quad \text { and } \quad \delta:=S^{1 / 2}-S^{-1 / 2},
$$

and

$$
\mathscr{Q}(\boldsymbol{\rho}, \grave{\rho}):=I_{0}(\boldsymbol{\rho} . \grave{\rho})=\left(\begin{array}{c}
\rho_{1} \grave{\rho}_{1} \\
0
\end{array}\right)
$$

\subsubsection{Symmetries}

We observe several useful properties of the mapping $\mathscr{G}_{c}$. If $f$ is even and $g$ is odd, then $A f$ and $\delta g$ are even, while $A g$ and $\delta f$ are odd. It follows that if $\rho_{1}$ is even and $\rho_{2}$ is odd, then $\mathscr{G}_{c}(\boldsymbol{\rho}, \mu) \cdot \mathbf{e}_{1}$ is even and $\mathscr{G}_{c}(\boldsymbol{\rho}, \mu) \cdot \mathbf{e}_{2}$ is odd, where $\mathbf{e}_{1}=(1,0)$ and $\mathbf{e}_{2}=(0,1)$. Next, $A f$ and $\delta f$ are "mean-zero" in the sense that if $f$ has a Fourier transform $\widehat{f}$, then $\widehat{A f}(0)=\widehat{\delta f}(0)=0$. Consequently, $\mathscr{G}_{c} \widehat{(\boldsymbol{\rho}, \mu)} \cdot \mathbf{e}_{1}(0)=0$, as well. We conclude that $\mathscr{G}_{c}$ maps

$$
\begin{aligned}
& \text { \{even functions }\} \times\{\text { odd functions }\} \times \mathbb{R} \\
& \quad \rightarrow \text { even mean-zero functions }\} \times\{\text { odd functions }\}
\end{aligned}
$$

\subsubsection{The $\mu=0$ Limit}

We are interested solutions to the traveling wave problem (2.3) in the limit as $\mu \rightarrow 0^{+}$, so we take $\mu=0$ and find that (2.3) reduces to the system

$$
\left\{\begin{array}{l}
c^{2} \rho_{1}^{\prime \prime}-A\left(\rho_{1}+\rho_{1}^{2}\right)+\kappa \delta \rho_{2}=0 \\
\kappa \rho_{2}=0
\end{array}\right.
$$

The second equation clearly has the solution $\rho_{2}=0$, at which point the first equation becomes

$$
c^{2} \rho_{1}^{\prime \prime}-A\left(\rho_{1}+\rho_{1}^{2}\right)=0 .
$$

This is the advance-delay differential equation that traveling wave profiles for the monatomic FPUT lattice must satisfy, and by Hypothesis 1 it has the solution $\rho=\sigma_{c}$.

\subsection{Linearizing at the Monatomic Solution}

To perturb from $\sigma_{c}$, we make the ansatz

$$
\rho=\sigma_{c}+\varrho
$$

for some small, exponentially localized $\varrho=\left(\varrho_{1}, \varrho_{2}\right)$. We then rearrange the equation $\mathscr{G}_{c}\left(\sigma_{c}+\right.$ $\varrho, \mu)=0$ into the system

$$
\left\{\begin{array}{l}
\mathscr{H}_{c} \varrho_{1}=\mathscr{R}_{1}(\rho, \mu) \\
\mathscr{L}_{\mu} \varrho_{2}=\mathscr{R}_{2}(\rho, \mu) .
\end{array}\right.
$$


The operator $\mathscr{H}_{c}$ was defined in (1.7); the new operator $\mathscr{L}_{\mu}$ is given by

$$
\mathscr{L}_{\mu} f:=c^{2} \mu f^{\prime \prime}+\kappa(1+\mu) f .
$$

The terms on the right side, absorbed into $\mathscr{R}_{c, 1}^{\mu}$ and $\mathscr{R}_{c, 2}^{\mu}$, are all "small" in that up to some $\mathscr{O}(1)$ linear operators, they are $\mu, \mu \varrho$, or $\varrho^{.2}=\left(\varrho_{1}, \varrho_{2}\right)$. The precise forms of these operators $\mathscr{R}_{c, 1}^{\mu}$ and $\mathscr{R}_{c, 2}^{\mu}$ are not important, as we will not work with exactly these terms in the future. Hypothesis 1 provides the invertibility of $\mathscr{H}_{C}$, so we obtain a fixed point equation for $\varrho_{1}$ easily:

$$
\varrho_{1}=\mathscr{H}_{c}^{-1} \mathscr{R}_{1}(\rho, \mu) .
$$

However, the operator $\mathscr{L}_{\mu}$ is not invertible. It is a singularly perturbed, constant coefficient second-order linear differential operator, and so we know that

$$
\mathscr{L}_{\mu} \sin \left(\Omega_{\mu} \cdot\right)=\mathscr{L}_{\mu} \cos \left(\Omega_{\mu} \cdot\right)=0,
$$

where

$$
\Omega_{\mu}:=\sqrt{\frac{\kappa(1+\mu)}{c^{2} \mu}} .
$$

We can also view $\mathscr{L}_{\mu}$ as a Fourier multiplier in the sense that

$$
\widehat{\mathscr{L}_{\mu} f}(k)=\left(-c^{2} k^{2}+\kappa(1+\mu)\right) \widehat{f}(k), f \in H_{q}^{2} .
$$

Importantly, the symbol of $\mathscr{L}_{\mu}$ vanishes at $k= \pm \Omega_{\mu}$, and so

$$
\widehat{\mathscr{L}_{\mu} f}\left( \pm \Omega_{\mu}\right)=0 .
$$

Consequently, $\mathscr{L}_{\mu}$ cannot be surjective as an operator $O_{q}^{r+2} \rightarrow O_{q}^{r}$, and so we have no hope of inverting $\mathscr{L}_{\mu}$ and converting the second equation in (2.8) into a tractable fixed point problem for $\varrho_{2}$.

However, while the solvability condition (2.12) impedes our progress with a classical perturbation attempt to solve the traveling wave problem, it will indicate a different method of resolution. First, $\mathscr{L}_{\mu}$ is injective, and we can estimate its inverse's operator norm relative to $\mu$.

Proposition 2 Let $r \geq 0$ and $\mu, q>0$ and set

$$
\mathfrak{D}_{\mu, q}^{r}:=\left\{f \in O_{q}^{r} \mid \widehat{f}\left(\Omega_{\mu}\right)=0\right\} .
$$

Then $\mathscr{L}_{\mu}$ is invertible from $O_{q}^{r+2}$ to $\mathfrak{D}_{\mu, q}^{r}$. Denoting the inverse operator from $\mathfrak{D}_{\mu, q}^{r}$ to $O_{q}^{r+2}$ by $\mathscr{L}_{\mu}^{-1}$, we have

$$
\left\|\mathscr{L}_{\mu}^{-1}\right\|_{\mathbf{B}\left(\mathfrak{D}_{\mu, q}^{r}, O_{q}^{r+j}\right)} \leq C(q) \mu^{-(1+j) / 2}, j=0,1,2,
$$

where $C(q)$ is independent of $\mu$ and $r$, but $C(q) \rightarrow \infty$ as $q \rightarrow 0^{+}$.

The proof of this proposition is in Appendix 1. Next, we can use (2.12) to characterize the range of $\mathscr{L}_{\mu}$. The pointwise evaluation of an exponentially localized function's Fourier transform at the critical value $\Omega_{\mu}$ as in (2.12) will be an essential linear functional for our future study. We name it and estimate it in the next proposition, whose proof is the same as that of Lemma A.5 in [14], which in turn is a modification of the classical Riemann-Lebesgue estimate for the Fourier transform. 
Proposition 3 For $g \in O_{q}^{r}$, let

$$
\iota_{\mu}[g]:=\int_{-\infty}^{\infty} g(x) \sin \left(\Omega_{\mu} x\right) d x .
$$

Then for all $q, r>0$, there is $C(q, r)>0$ such that if $f \in O_{q}^{r}$, then

$$
\left|\iota_{\mu}[f]\right| \leq C(q, r) \mu^{r / 2} .
$$

Using the language of the preceding two propositions, we can then characterize the range of $\mathscr{L}_{\mu}$ via

$$
\mathscr{L}_{\mu} f=g, f \in O_{q}^{r+2}, g \in O_{q}^{r} \Longleftrightarrow \iota_{\mu}[g]=0 .
$$

In the next section, we discuss how this solvability condition motivates a revised traveling wave ansatz, the nanopteron.

\subsection{The Nanopteron Strategy}

The traveling wave problem (2.8) is overdetermined: we must solve the two equations $\varrho_{1}=$ $\mathscr{H}_{c}^{-1} \mathscr{R}_{1}(\varrho, \mu)$ and $\mathscr{L}_{c} \varrho_{2}=\mathscr{R}_{2}(\varrho, \mu)$, while the solvability condition (2.16) demands that the equation $\iota_{\mu}\left[\mathscr{R}_{2}(\varrho, \mu)\right]=0$ be met as well. A very similar situation arose in the long wave problem for diatomic FPUT lattices [14]. There, the traveling wave problem contained two unknowns, like our $\varrho_{1}$ and $\varrho_{2}$, but a third equation, like (2.16), had to be met. And, like our (2.16), the third equation in the long wave problem involved a functional of the form "integrate against a sinusoid."

Similar solvability conditions arise in the spring dimer long wave problem [11], and a long wave problem for the capillary-gravity Whitham equation, studied by Johnson and Wright [29]. Like the prototypical model problem of Amick and Toland [1], all of these solvability conditions hinge on the vanishing of an "integrate against a sinusoid" functional. Substantially more complicated solvability conditions appear in the small mass FPUT problem [25] and in the equal mass FPUT problem [13]. Both still require a functional of the form "integrate against a function $\gamma$ " to vanish, but the precise function $\gamma$ is no longer an exact sinusoid. For the small mass FPUT limit, $\gamma$ is an asymptotically sinusoidal "Jost solution" of a small perturbation of a Schrodinger operator by nonlocal terms, while in the equal mass limit $\gamma$ is an asymptotically sinusoidal kernel element of an advance-delay differential operator that has exponentially localized, variable coefficient terms. In the small mass and equal mass FPUT problems, the chief technical challenge (and accomplishment) is, arguably, the construction of the function $\gamma$ that gives the precise solvability condition.

But despite the differences in the exact form of the solvability condition, all of these problems employ a related technique, originally due to Beale's work on capillary-gravity water waves [3] and subsequently refined by Amick and Toland for a simpler model problem. Here is the chief idea. The linearization of our putative fixed point problem

$$
\left\{\begin{array}{l}
\varrho_{1}=\mathscr{H}_{c}^{-1} \mathscr{R}_{1}(\varrho, \mu) \\
\mathscr{L}_{c} \varrho_{2}=\mathscr{R}_{2}(\varrho, \mu)
\end{array}\right.
$$

is the diagonal operator $\operatorname{diag}\left(\mathbb{1}, \mathscr{L}_{\mu}\right)$, and, by $(2.10)$, we see that this operator annihilates the sinusoidal functions $\left(0, \sin \left(\Omega_{\mu^{*}}\right)\right)$ and $\left(0, \cos \left(\Omega_{\mu^{*}}\right)\right)$, where $\Omega_{\mu}$ is defined in (2.11). The operator $\operatorname{diag}\left(\mathbb{1}, \mathscr{L}_{\mu}\right)$ is injective from $E_{q}^{r+2} \times O_{q}^{r+2}$ to $E_{q}^{r} \times O_{q}^{r}$ but not surjective due to (2.16). 
Beale encountered a similar obstacle and first observed that these exact sinusoidal solutions to the linearization extend to exact periodic solutions of the full nonlinear problem (2.17). The same is true for our small mass MiM problem. That is, there are solutions to (2.17) of the form $\rho=a \phi_{\mu}^{a}$ for $\mu$ and $a$ small, where $\phi_{\mu}^{a}$ is periodic and $a$ is, roughly, an "amplitude parameter." We prove this in Theorem 2. Beale then modified his perturbation ansatz to incorporate these periodic terms, and we do the same, replacing (2.7) with

$$
\rho=\sigma_{c}+a \phi_{\mu}^{a}+\eta
$$

where $\eta=\left(\eta_{1}, \eta_{2}\right) \in E_{q}^{r} \times O_{q}^{r}$ for appropriate $q$ and $r$. We then have three unknowns- $a$, $\eta_{1}$, and $\eta_{2}$ - and we parley the two equations in (2.17) and the solvability condition into three fixed point equations for these three unknowns. This is the content of most of Sect. 4. The construction of an equation for $\eta_{1}$ is easy, as it is effectively given already in fixed point form as the first equation in (2.17). In Proposition 4 we spell out exactly how one exploits the solvability condition in conjunction with the second equation in (2.17) to summon the separate equations for $\eta_{2}$ and $a$. This is all in line with the spirit of nanopteron constructions past in $[1,11,13,14,29]$.

\subsection{A Refined Leading Order Limit}

Before proceeding, we need to change slightly the monatomic solution from which we perturb. An easy calculation shows

$$
\mathscr{G}_{c}\left(\sigma_{c}, \mu\right)=\left(\begin{array}{c}
0 \\
-\mu \delta\left(\sigma_{c}+\sigma_{c}^{2}\right)
\end{array}\right),
$$

and so $\sigma_{c}$ solves $\mathscr{G}_{c}(\rho, \mu)=0$ to $\mathscr{O}(\mu)$. It turns out that we can, and in fact must, do better. We will find a function $\zeta_{c}^{\mu}$ that is an $\mathscr{O}(\mu)$ perturbation of $\sigma_{c}$ and that solves $\mathscr{G}_{c}(\boldsymbol{\rho}, \mu)=0$ to $\mathscr{O}\left(\mu^{2}\right)$. We will then replace $\sigma_{c}$ in our perturbation ansatz by $\boldsymbol{\zeta}_{c}^{\mu}$. The need to make this "refined leading order limit," which was also necessary in the small mass FPUT problem, arises for very technical reasons deep in the appendices; see Remark 2 for a discussion much later in the right context of why this change matters.

Here is our result, which we prove using the implicit function theorem, exactly as Hoffman and Wright establish Lemma 4.1 in their paper.

Lemma 1 There exists $\mu_{\varsigma}>0$ such that for all $\mu \in\left(0, \mu_{\varsigma}\right)$, there is $\zeta_{c}^{\mu}=\left(\zeta_{c, 1}^{\mu}, \zeta_{c, 2}^{\mu}\right) \in$ $\cap_{r=0}^{\infty} E_{q_{c}}^{r} \times O_{q_{c}}^{r}$ such that if

$$
\boldsymbol{\zeta}_{c}^{\mu}=\left(\begin{array}{c}
\varsigma_{c, 1}^{\mu} \\
\varsigma_{c, 2}^{\mu}
\end{array}\right):=\sigma_{c}+\mu \zeta_{c}^{\mu},
$$

then $\mathscr{G}_{c}\left(\boldsymbol{\zeta}_{c}^{\mu}, \mu\right) \cdot \mathbf{e}_{1}=0$ and $\mathscr{G}_{c}\left(\boldsymbol{\zeta}_{c}^{\mu}, \mu\right) \cdot \mathbf{e}_{2}=\mathscr{O}\left(\mu^{2}\right)$. Moreover, for each $r \geq 0$,

$$
\sup _{\mu \in\left(0, \mu_{\zeta}\right)}\left\|\zeta_{c}^{\mu}\right\|_{r, q_{c}} \leq C(r) .
$$

\section{Periodic Solutions}

In this section we state a theorem on the existence of periodic solutions to our traveling wave problem (2.3). First, we rescale our traveling wave profiles $\rho$ to

$$
\rho(x)=I^{\mu} \boldsymbol{\phi}(\omega x),
$$


where $I^{\mu}:=\operatorname{diag}(\mu, 1), \phi(X)=\left(\phi_{1}(X), \phi_{2}(X)\right)$ is $2 \pi$-periodic, and $\omega \in \mathbb{R}$. We assume $\phi \in E_{\mathrm{per}, 0}^{2} \times O_{\mathrm{per}}^{2}$, where

$$
\begin{aligned}
& H_{\mathrm{per}}^{r}=\left\{f \in L_{\text {per }}^{2} \mid\|f\|_{r}<\infty\right\}, \quad\langle f, g\rangle_{r}:=\sum_{k=-\infty}^{\infty}\left(1+k^{2}\right)^{r} \widehat{f}(k) \widehat{\widehat{g}}(k), \quad\|f\|_{r}:=\sqrt{\langle f, f\rangle_{r}}, \\
& E_{\text {per }}^{r}=H_{\text {per }}^{r} \cap\{\text { even functions }\}, \\
& E_{\text {per }, 0}^{r}=\left\{f \in E_{\text {per }}^{r} \mid \widehat{f}(0)=0\right\},
\end{aligned}
$$

and

$$
O_{\text {per }}^{r}=H_{\text {per }}^{r} \cap\{\text { odd functions }\} .
$$

Inserting the periodic ansatz (3.1) into (2.3), we find $\mathscr{G}_{c}\left(I^{\mu} \boldsymbol{\phi}(\omega \cdot), \mu\right)=0$ if and only if

$$
\underbrace{c^{2} \mu \omega^{2} \boldsymbol{\phi}^{\prime \prime}+\mathscr{D}_{\mu}[\omega] I^{\mu} \boldsymbol{\phi}+\mathscr{D}_{\mu}[\omega] \mathscr{Q}\left(I^{\mu} \boldsymbol{\phi}, I^{\mu} \boldsymbol{\phi}\right)}_{\boldsymbol{\Phi}_{\mu}(\boldsymbol{\phi}, \omega)}=0 .
$$

Here we define $\mathscr{D}_{\mu}[\omega]$ by replacing any shift operator $S^{d}$ in the definition of $\mathscr{D}_{\mu}$ in (2.4) with $S^{d \omega}$. We will construct solutions to this periodic problem (3.2) using a modification of the "bifurcation from a simple eigenvalue" argument of Crandall and Rabinowitz [7] and Zeidler [53] that has already been deployed successfully in the FPUT long wave (Theorem 4.1 in [14] and Theorem 3.1 in [11]), small mass (Theorem 5.1 in [25]), and equal mass (Proposition 3.3 in [13]) problems. The analysis takes place chiefly at the linear level.

First we examine the kernel of the linearization of (3.2), and so we study the equation

$$
\underbrace{c^{2} \mu \omega^{2} \boldsymbol{\phi}^{\prime \prime}+\mathscr{D}_{\mu}[\omega] I^{\mu} \boldsymbol{\phi}}_{\Gamma_{\mu}[\omega] \boldsymbol{\phi}}=0 .
$$

Viewed on the Fourier side, a solution $\phi$ to $\Gamma_{\mu}[\omega] \phi=0$ must satisfy

$$
c^{2} \mu \omega^{2} k^{2} \widehat{\boldsymbol{\phi}}(k)=\widetilde{\mathscr{D}}_{\mu}[\omega k] I^{\mu} \widehat{\boldsymbol{\phi}}(k), k \in \mathbb{Z},
$$

where $\widetilde{\mathscr{D}}_{\mu}[K] \in \mathbb{C}^{2 \times 2}$ is the matrix

$$
\widetilde{\mathscr{D}}_{\mu}[K]:=\left[\begin{array}{cc}
-(2 \cos (K)-2) & 2 i \kappa \sin \left(\frac{K}{2}\right) \\
-2 i \mu \sin \left(\frac{K}{2}\right) & \kappa(1+\mu)
\end{array}\right] .
$$

If $\widehat{\boldsymbol{\phi}}(k) \neq 0$, then (3.3) shows that $c^{2} \mu \omega^{2} k^{2}$ must be an eigenvalue of $\widetilde{\mathscr{D}}_{\mu}[\omega k] I^{\mu}$ with eigenvector $\widehat{\boldsymbol{\phi}}(k)$. We compute that the eigenvalues of $\widetilde{\mathscr{D}}_{\mu}[K]$ are

$$
\lambda_{\mu}^{ \pm}(K):=\frac{\kappa}{2}+\mu\left(\frac{\kappa}{2}+1-\cos (K)\right) \pm \frac{\sqrt{P_{\mu}(\cos (K))}}{2},
$$

where

$$
P_{\mu}(t):=(\kappa-2 t+2)^{2} \mu^{2}+2 \kappa(\kappa+2 t-2) \mu+\kappa^{2} .
$$

So, if (3.3) holds with $\widehat{\phi}(k) \neq 0$, we have either

$$
c^{2} \mu \omega^{2} k^{2}=\lambda_{\mu}^{-}(\omega k) \quad \text { or } \quad c^{2} \mu \omega^{2} k^{2}=\lambda_{\mu}^{+}(\omega k)
$$


We will show that the first equality never holds, assuming $\phi \in E_{\mathrm{per}, 0}^{2} \times O_{\mathrm{per}}^{2}$, while the second holds only when $\omega=\omega_{\mu} / k$, where $k \in \mathbb{Z} \backslash\{0\}$ and $\omega_{\mu}$ is an $\mathscr{O}\left(\mu^{3 / 2}\right)$ perturbation of the original critical frequency $\Omega_{\mu}$ from (2.11). Specifically, $\omega_{\mu}$ has the following properties.

Lemma 2 Let $|c|>1$.

(i) There is $\mu_{\omega}>0$ such that if $0<\mu<\mu_{\omega}$, there is a unique $\omega_{\mu}>0$ such that

$$
c^{2} \omega_{\mu}^{2}-\lambda_{\mu}^{+}\left(\omega_{\mu}\right)=0 .
$$

(ii) $\omega_{\mu}=\mathscr{O}\left(\mu^{-1 / 2}\right)$. More precisely,

$$
\Omega_{\mu}=\frac{\sqrt{\kappa(1+\mu)}}{c \sqrt{\mu}} \leq \omega_{\mu} \leq \frac{\sqrt{\lambda_{\mu}^{+}(\pi)}}{c \sqrt{\mu}} .
$$

(iii) $\omega_{\mu}-\Omega_{\mu}=\mathscr{O}\left(\mu^{3 / 2}\right)$.

We use these properties of $\omega_{\mu}$ in a bifurcation argument in Appendix B to prove the following theorem.

Theorem 2 Suppose $|c|>1$. There is $\mu_{\mathrm{per}} \in\left(0, \mu_{\omega}\right)$ and $a_{\mathrm{per}}>0$ such that for all $0<\mu<$ $\mu_{\text {per }}$ there are maps

$$
\begin{aligned}
& {\left[-a_{\text {per }}, a_{\text {per }}\right] \rightarrow \mathbb{R}: a \mapsto \omega_{\mu}^{a}} \\
& {\left[-a_{\text {per }}, a_{\text {per }}\right] \rightarrow \mathscr{C}_{\text {per }}^{\infty} \cap\{\text { even functions }\}: a \mapsto \psi_{\mu, 1}^{a}} \\
& {\left[-a_{\text {per }}, a_{\text {per }}\right] \rightarrow \mathscr{C}_{\text {per }}^{\infty} \cap\{\text { odd functions }\}: a \mapsto \psi_{\mu, 2}^{a}}
\end{aligned}
$$

with the following properties.

(i) If

$$
\boldsymbol{\phi}_{\mu}^{a}(x)=\left(\begin{array}{c}
\phi_{\mu, 1}^{a}(x) \\
\phi_{\mu, 2}^{a}(x)
\end{array}\right):=\left(\begin{array}{c}
\mu v_{\mu} \cos \left(\omega_{\mu}^{a} x\right) \\
\sin \left(\omega_{\mu}^{a} x\right)
\end{array}\right)+\left(\begin{array}{c}
\mu \psi_{\mu, 1}^{a}\left(\omega_{\mu}^{a} x\right) \\
\psi_{\mu, 2}^{a}\left(\omega_{\mu}^{a} x\right)
\end{array}\right),
$$

where

$$
v_{\mu}=\sin \left(\frac{\omega_{\mu}}{2}\right) \alpha_{\mu}
$$

and $\alpha_{\mu}=\mathscr{O}(1)$ is defined below in (B.14), then $\mathscr{G}_{c}\left(a \phi_{\mu}^{a}, \mu\right)=0$.

(ii) $\omega_{\mu}^{0}=\omega_{\mu}$.

(iii) $\psi_{\mu, 1}^{0}=\psi_{\mu, 2}^{0}=0$.

(iv) For all $r \geq 0$, there is $C_{r}>0$ such that if $|a|,|\grave{a}| \leq a_{\mathrm{per}}$, then

$$
\left|\omega_{\mu}^{a}-\omega_{\mu}^{\grave{a}}\right|+\left\|\psi_{\mu, 1}^{a}-\psi_{\mu, 1}^{\grave{a}}\right\|_{W^{r, \infty}}+\left\|\psi_{\mu, 2}^{a}-\psi_{\mu, 2}^{\grave{a}}\right\|_{W^{r, \infty}} \leq C_{r}|a-\grave{a}| .
$$

\section{The Nanopteron Problem}

\subsection{Beale's ansatz}

We evaluate the traveling wave problem (2.3) at Beale's ansatz

$$
\rho=\boldsymbol{\varsigma}_{c}+a \boldsymbol{\phi}_{\mu}^{a}+\eta,
$$

where $|\mu|<\min \left\{\mu_{\varsigma}, \mu_{\text {per }}\right\}$ and 
- The function $\boldsymbol{\zeta}_{c}=\sigma_{c}+\mu \zeta_{c}^{\mu}$ is our refined leading order limit from Theorem 1, with $\mathscr{G}_{c}\left(\boldsymbol{\zeta}_{c}, \mu\right) \cdot \mathbf{e}_{1}=0$ and $\mathscr{G}_{c}\left(\boldsymbol{\zeta}_{c}, \mu\right) \cdot \mathbf{e}_{2}=\mathscr{O}\left(\mu^{2}\right)$;

- The functions $a \phi_{\mu}^{a},|a| \leq a_{\mathrm{per}}$, are the family of periodic solutions from Theorem 2, which satisfy $\mathscr{G}_{c}\left(a \boldsymbol{\phi}_{\mu}^{a}, \mu\right)=0$;

- And $\eta=\left(\eta_{1}, \eta_{2}\right) \in E_{q}^{r} \times O_{q}^{r}$, with $q$ and $r$ to be determined later.

After inserting Beale's ansatz (4.1) into the traveling wave problem, we use the linearity of $\mathscr{D}_{\mu}$, the bilinearity of $\mathscr{Q}$, and the properties of the terms of the ansatz stated above to obtain the equation

$c^{2} I_{\mu} \boldsymbol{\eta}^{\prime \prime}+\mathscr{D}_{\mu} \boldsymbol{\eta}+2 \mathscr{D}_{\mu} \mathscr{Q}\left(\boldsymbol{\zeta}_{c}, \boldsymbol{\eta}\right)=-\mathscr{G}_{c}\left(\boldsymbol{\zeta}_{c}, \mu\right)-2 a \mathscr{D}_{\mu} \mathscr{Q}\left(\boldsymbol{\zeta}_{c}, \boldsymbol{\phi}_{\mu}^{a}\right)-2 a \mathscr{D}_{\mu} \mathscr{Q}\left(\boldsymbol{\phi}_{\mu}^{a}, \boldsymbol{\eta}\right)-\mathscr{D}_{\mu} \mathscr{Q}(\boldsymbol{\eta}, \boldsymbol{\eta})$.

Now we expose the components of this system. We use the definitions of $\mathscr{D}_{\mu}$ and $\mathscr{Q}$ from (2.4) and (2.5), respectively, and the results that $\mathscr{G}_{c}\left(a \boldsymbol{\phi}_{\mu}^{a}, \mu\right)=\mathscr{G}_{c}\left(\boldsymbol{\zeta}_{c}, \mu\right) \cdot \mathbf{e}_{1}=0$ to rewrite (4.2) as

$$
\left\{\begin{array}{l}
\mathscr{H}_{c} \eta_{1}=h_{1}^{\mu}(\boldsymbol{\eta}, a)+h_{2}^{\mu}(\boldsymbol{\eta}, a)+h_{3}^{\mu}(\boldsymbol{\eta}, a)+h_{4}^{\mu}(\boldsymbol{\eta}, a)-\kappa \delta \eta_{2} \\
\mathscr{L}_{\mu} \eta_{2}=\ell_{1}^{\mu}(\boldsymbol{\eta}, a)+\ell_{2}^{\mu}(\boldsymbol{\eta}, a)+\ell_{3}^{\mu}(\boldsymbol{\eta}, a)+2 a \mu \delta\left[\varsigma_{c, 1}^{\mu} \phi_{\mu, 1}^{a}\right],
\end{array}\right.
$$

where

$$
\begin{aligned}
h_{1}^{\mu}(\boldsymbol{\eta}, a) & :=2 \mu A\left(\zeta_{c, 1}^{\mu} \eta_{1}\right) & & \ell_{1}^{\mu}(\boldsymbol{\eta}, a):=-\mathscr{G}_{c}\left(\boldsymbol{\zeta}_{c}, \mu\right) \cdot \mathbf{e}_{2} \\
h_{2}^{\mu}(\boldsymbol{\eta}, a) & :=2 a A\left(\varsigma_{c, 1}^{\mu} \phi_{\mu, 1}^{a}\right) & & \ell_{2}^{\mu}(\boldsymbol{\eta}, a):=\mu \delta\left[\eta_{1}+\varsigma_{c, 1}^{\mu} \eta_{1}+\eta_{1}^{2}\right] \\
h_{3}^{\mu}(\boldsymbol{\eta}, a) & :=2 a A\left(\phi_{\mu, 1}^{a} \eta_{1}\right) & & \ell_{3}^{\mu}(\boldsymbol{\eta}, a):=2 a \mu \delta\left(\phi_{\mu, 1}^{a} \eta_{1}\right) \\
h_{4}^{\mu}(\boldsymbol{\eta}, a) & :=A\left(\eta_{1}^{2}\right) . & &
\end{aligned}
$$

The operators $\mathscr{H}$ and $\mathscr{L}_{\mu}$ are the same as they were in (1.7) and (2.9) from our initial attempt at a perturbation problem. One should view the seven terms $h_{1}^{\mu}, h_{2}^{\mu}, h_{3}^{\mu}, \ell_{1}^{\mu}, \ldots, \ell_{4}^{\mu}$ as "small." For example, $h_{1}^{\mu}$ is roughly $\mu \eta_{1}, h_{2}^{\mu}$ is roughly $\mu a$, when we recall from (3.8) that $\phi_{\mu, 1}^{a}$ bears a factor of $v_{\mu}=\mathscr{O}(\mu)$, and $h_{3}^{\mu}$ and $h_{4}^{\mu}$ are quadratic in $a$ and $\mu$. We choose to leave the last terms in the $\eta_{1}$ and $\eta_{2}$ equations explicit for now, as they will require more modifications shortly.

\subsection{The Fixed Point Equations for $\eta_{2}$ and a: Motivation}

We motivate our construction of these equations by thinking about how the inverse function theorem could apply to our situation. The second equation in (4.3) reads

$$
\mathscr{L}_{\mu} \eta_{2}=\underbrace{\sum_{k=1}^{3} \ell_{k}^{\mu}(\boldsymbol{\eta}, a)+2 a \mu \delta\left[\varsigma_{c, 1}^{\mu} \phi_{\mu, 1}^{a}\right]}_{\mathscr{R}_{\mu}(\boldsymbol{\eta}, a)} .
$$

If this is to hold, the solvability condition (2.16) requires

$$
\iota_{\mu}\left[\mathscr{R}_{\mu}(\boldsymbol{\eta}, a)\right]=0 .
$$


The inverse function theorem will permit us to solve (4.5) for $a$ in terms of $\eta$ if

$$
\left.\frac{\partial}{\partial a} \iota_{\mu}\left[\sum_{k=1}^{3} \ell_{k}^{\mu}(\boldsymbol{\eta}, a)+2 a \mu \delta\left[\varsigma_{c, 1}^{\mu} \phi_{\mu, 1}^{a}\right]\right]\right|_{\boldsymbol{\eta}=0, a=0}=2 \mu \iota_{\mu}\left[\delta\left[\varsigma_{c, 1}^{\mu} \phi_{\mu, 1}^{0}\right]\right] \neq 0
$$

This calculation holds because the terms $\ell_{1}^{\mu}$ and $\ell_{2}^{\mu}$ are constant in $a$, while $\ell_{3}^{\mu}$ is quadratic in $a$ and $\eta$; this calculation, though, is purely formal because we never established the differentiability of the map $a \mapsto \boldsymbol{\phi}_{\mu}^{a}$ as part of Theorem 2. And so we will not use the inverse function theorem here, not least because we would require further results on the periodic functions, but also because we would need some highly quantitative modification of that classical theorem to handle the $\mu$ parameter.

Instead, we modify the second equation in (4.3) based on what (4.6) suggests the leading order term in $a$ should be. Observe that

$$
\begin{aligned}
2 a \mu \delta\left[\zeta_{c, 1}^{\mu} \phi_{\mu, 1}^{a}\right]= & 2 a \mu \delta\left[\left(\sigma_{c}+\mu \zeta_{c, 1}^{\mu}\right) \phi_{\mu, 1}^{a}\right]=2 a \mu \delta\left[\sigma_{c} \phi_{\mu, 1}^{0}\right] \\
& +\underbrace{2 a \mu \delta\left[\sigma_{c}\left(\phi_{\mu, 1}^{a}-\phi_{\mu, 1}^{0}\right)\right]}_{\ell_{4}^{\mu}(\boldsymbol{\eta}, a)}+\underbrace{2 a \mu^{2} \delta\left(\zeta_{c, 1}^{\mu} \phi_{\mu, 1}^{a}\right)}_{\ell_{5}^{\mu}(\boldsymbol{\eta}, a)} .
\end{aligned}
$$

We think of $\ell_{4}^{\mu}$ as being roughly quadratic in $a$, while $\ell_{5}^{\mu}$ has the very small factor $\mu^{2}$.

Theorem 2 allows us to calculate

$$
\phi_{\mu, 1}^{0}=\mu v_{\mu} \cos \left(\omega_{\mu} \cdot\right)
$$

where $v_{\mu}=\mathscr{O}(1)$ is defined in (3.9). Recall from Lemma 2 that $\omega_{\mu}$ is an $\mathscr{O}\left(\mu^{3 / 2}\right)$ perturbation of the original critical frequency $\Omega_{\mu}$, defined in (2.11). For reasons that will be clearer in the proper context later, specifically Remark 1, we want to make one further adjustment to permit $\Omega_{\mu}$ to play more of a starring role. We rewrite

$$
2 a \mu \delta\left[\sigma_{c} \phi_{\mu, 1}^{0}\right]=\underbrace{2 a \mu^{2} v_{\mu} \delta\left[\sigma_{c} \cos \left(\Omega_{\mu} \cdot\right)\right]}_{-a \chi_{\mu}}+\underbrace{2 a \mu^{2} v_{\mu} \delta\left[\sigma_{c}\left(\cos \left(\omega_{\mu} \cdot\right)-\cos \left(\Omega_{\mu} \cdot\right)\right)\right]}_{\ell_{6}^{\mu}(\boldsymbol{\eta}, a)} .
$$

That is, we have set

$$
\chi_{\mu}:=-2 \mu^{2} v_{\mu} \delta\left[\sigma_{c} \cos \left(\Omega_{\mu} \cdot\right)\right] .
$$

Combining (4.7) and (4.8), we see that the second equation in (4.3) is equivalent to

$$
\mathscr{L}_{\mu} \eta_{2}+a \chi_{\mu}=\sum_{k=1}^{6} \ell_{k}^{\mu}(\eta, a)
$$

We pause to isolate some precise estimates on $\chi_{\mu}$.

Lemma 3 For each $r \geq 0, \mu \in\left(0, \mu_{\mathrm{per}}\right)$, and $q \in\left[0, q_{c}\right]$, we have $\chi_{\mu} \in E_{q}^{r}$, and there is $C(q, r)>0$ such that

$$
\left\|\chi_{\mu}\right\|_{r, q} \leq C(q, r) \mu^{2-r / 2}
$$


Now we sketch how we obtain the equation for $a$. Recall that the functional $\iota_{\mu}$, defined in (2.14), annihilates the range of $\mathscr{B}_{\mu}$. Applying this functional to (4.10), we have

$$
a \iota_{\mu}\left[\chi_{\mu}\right]=\sum_{k=1}^{6} \iota_{\mu}\left[\ell_{k}^{\mu}(\eta, a)\right] .
$$

Consequently, we want to write

$$
a=\frac{1}{\iota_{\mu}\left[\chi_{\mu}\right]} \sum_{k=1}^{6} \iota_{\mu}\left[\ell_{k}^{\mu}(\eta, a)\right] .
$$

Of course, this is valid only if $\iota_{\mu}\left[\chi_{\mu}\right] \neq 0$.

In Appendix C.2, we establish the following identity for $\chi_{\mu}$ :

$$
\iota_{\mu}\left[\chi_{\mu}\right]=\mu^{2} \sin ^{2}\left(\frac{\Omega_{\mu}}{2}\right) \alpha_{\mu}\left\|\sigma_{c}\right\|_{L^{1}}+\mathscr{O}\left(\mu^{7 / 2}\right),
$$

where $\alpha_{\mu}=\mathscr{O}(1)$ is defined precisely in (B.14) and $\left\|\sigma_{c}\right\|_{L^{1}}>0$. So, the denominator in (4.12) is at least $\mathscr{O}\left(\mu^{2}\right)$, which is small.

However, the denominator can be smaller, for it is possible to have $\sin \left(\Omega_{\mu} / 2\right)=0$. Specifically, from the definition of $\Omega_{\mu}$ in (2.11) this happens if and only if

$$
\mu=\frac{\kappa}{4 \pi^{2} n^{2} c^{2}-\kappa}=: \mu_{n}, n \in \mathbb{N} .
$$

One can check that these $\mu_{n}$ are precisely the "antiresonance" mass ratios that Kevrekidis, Stefanov, and Xu encounter in [31] and for which they produce solitary traveling waves in their Hertzian MiM lattice; see Sect. 4.3 momentarily for a discussion of how these $\mu_{n}$ relate to solitary waves in our MiM lattice with cubic FPUT potential.

If we attempt to run our nanopteron program including these $\mu_{n}$, we will encounter an $\mathscr{O}\left(\mu^{-7 / 2}\right)$ factor in the equation (4.12) for $a$, and this is simply too large for any reasonable estimate. Another way to view this complication is that if $\mu=\mu_{n}$, then the formal derivative in (4.6) could be $\mathscr{O}\left(\mu^{7 / 2}\right)$, which is to say that it could be 0 , in which case the inverse function theorem fails to solve (4.5). So, we exclude these potentially pernicious values $\mu_{n}$ from consideration by restricting $\mu$ to the set

$$
\tilde{\mathbf{M}}_{c, \kappa}:=\left\{\mu \in\left(0, \mu_{\text {per }}\right)|| \sin \left(\frac{\Omega_{\mu}}{2}\right) \mid>\frac{1}{2}\right\} .
$$

Upon doing so, the formula (4.13) implies the next lemma.

Lemma 4 There exist $\mu_{\chi} \in\left(0, \mu_{\mathrm{per}}\right)$ and $C_{\chi}>0$ such that if $\mu \in \tilde{\mathrm{M}}_{c, \kappa} \cap\left(0, \mu_{\chi}\right)$, then

$$
C_{\chi} \mu^{2}<\left|\iota_{\mu}\left[\chi_{\mu}\right]\right| \text {. }
$$

Remark 1 Had we not added zero and made the expansion (4.8), all the subsequent calculations above would still go through, with $\Omega_{\mu}$ replaced by $\omega_{\mu}$ throughout. We would still need to restrict $\mu$ to a set like $\widetilde{\mathbf{M}}_{c, \kappa}$, as the continuity and unboundedness of the map $\mu \mapsto \omega_{\mu}$, which one can extract from (3.7), imply that $\sin \left(\omega_{\mu} / 2\right)=0$ for a countable number of $\mu$. However, we do not have an exact formula for $\omega_{\mu}$, only the implicit relation (3.7). So, working with the original critical frequency $\Omega_{\mu}$ and its accompanying antiresonance mass ratios $\mu_{n}$ is much more transparent. Furthermore, as we discuss in the next section, it is possible to divine the traveling wave behavior of the MiM lattice at these $\mu_{n}$, too. 


\subsection{Traveling Waves at the Antiresonance Values for $\mu$}

Hoffman and Wright also had to exclude a countable number of mass ratios $\mu$ from their nanopteron program in [25] for exactly the same reasons that led to our restriction to $\tilde{\mathrm{M}}_{c, \kappa}$. Such a restriction is not an egregious omission of small values of $\mu$; since $\kappa /\left((2 n+1)^{2} \pi^{2}-\right.$ $\kappa) \in \widetilde{\mathbf{M}}_{c, \kappa}$, we have $0 \in \widetilde{\mathbf{M}}_{c, \kappa}$. Moreover, the lower bound of $1 / 2$ in the definition of $\widetilde{\mathbf{M}}_{c, \kappa}$ is arbitrary; we could fix any $b \in(0,1)$ and replace $1 / 2$ with $b$, and still our nanopteron program would run smoothly (with $C_{\chi}$ now depending, arguably badly, on $b$ ).

All this is to say that, effectively, we can produce nanopteron traveling waves for $\mu$ arbitrarily close to the values $\mu_{n}$ from (4.14). But what happens when $\mu=\mu_{n}$ ? Our arguments here certainly do not extend to these values. It turns out that the MiM lattice bears genuine solitary traveling waves when $\mu=\mu_{n}$, just as the MiM lattice with Hertzian potential does. This is proved by Faver, Goodman, and Wright in the forthcoming [12].

The crux of their argument is that when $\mu=\mu_{n}$, it is possible to rewrite the original traveling wave problem (2.3) as a single equation in the unknown $\rho_{1}$, to which a more routine perturbation argument can be applied. This rewriting, in turn, hinges on viewing the second component of the traveling wave problem (2.3), which is

$$
\mathscr{L}_{\mu_{n}} \rho_{2}=\mu_{n} \delta\left(\rho_{1}+\rho_{1}^{2}\right)
$$

on the Fourier side as

$$
-\left(c^{2} \mu_{n} k^{2}-\kappa\left(1+\mu_{n}\right)\right) \widehat{\rho}_{2}(k)=2 i \mu_{n} \sin \left(\frac{k}{2}\right)\left(\widehat{\rho}_{1}+\widehat{\rho_{1}^{2}}\right) .
$$

The function

$$
\widetilde{\mathscr{M}}_{n}(k):=-\frac{2 i \mu_{n} \sin (k / 2)}{c^{2} \mu_{n} k^{2}-\kappa(1+\mu)}
$$

is analytic on $\mathbb{C}$ with removable singularities at $k= \pm \Omega_{\mu_{n}}$. Consequently, Fourier multiplier theory due to Beale (Lemma 7 in this paper) implies that the Fourier multiplier $\mathscr{M}_{n}$ with symbol $\widetilde{\mathscr{M}}_{n}$ maps $E_{q}^{r}$ to $E_{q}^{r+2}$, and so (4.17) is equivalent to

$$
\rho_{2}=\mathscr{M}_{n}\left(\rho_{1}+\rho_{1}^{2}\right) \text {. }
$$

Then one feeds this equation into the first component of (2.3) to obtain an equation solely in $\rho_{1}$. It is also possible to show that the operator norm of $\mathscr{M}_{n}$ is $\mathscr{O}(1 / n)$, and thereby hangs the perturbation argument. We emphasize, though, that the whole procedure very much depends on taking $\mu=\mu_{n}$ from (4.14), so that the zeros of the denominator of $\widetilde{\mathscr{M}}_{n}$ cancel with those of the numerator. This, we feel, justifies our insistence on making $\Omega_{\mu}$ explicit in the nanopteron program via (4.8).

\subsection{The Fixed Point Equations for $\eta_{2}$ and $a$ : Construction}

Now that we have controlled our permitted values of $\mu$, we are ready to state precisely how we extract two fixed point equations, one for $\eta_{2}$ and one for $a$, from the single equation (4.10).

Proposition 4 Let $q>0, r \geq 0$, and $\mu \in \widetilde{\mathbf{M}}_{c, \kappa} \cap\left(0, \mu_{\chi}\right)$. There exist a bounded linear functional $\mathscr{A}_{\mu}: O_{q}^{r} \rightarrow \mathbb{R}$ and a bounded operator $\mathscr{B}_{\mu}: O_{q}^{r} \rightarrow O_{q}^{r+2}$ such that, given $f \in O_{q}^{r+2}, a \in \mathbb{R}$, and $g \in O_{q}^{r}$, we have

$$
\mathscr{L}_{\mu} f+a \chi_{\mu}=g
$$


if and only if

$$
a=\mathscr{A}_{\mu} g \quad \text { and } \quad f=\mathscr{B}_{\mu} g .
$$

Moreover, the operators $\mathscr{A}_{\mu}$ and $\mathscr{B}_{\mu}$ satisfy

$\left\|\mathscr{A}_{\mu}\right\|_{\mathbf{B}\left(O_{q}^{r}, \mathbb{R}\right)} \leq C(q, r) \mu^{-2+r / 2} \quad$ and $\quad\left\|\mathscr{B}_{\mu}\right\|_{\mathbf{B}\left(O_{q}^{r}, O_{q}^{r+j}\right)} \leq C(q, r) \mu^{-(j+1) / 2}, j=0,1,2$.

Proof Suppose that (4.18) holds and apply the functional $\iota_{\mu}$ from (2.14) to both sides of this equation. Then we can use Lemma 4 to solve for $a$ :

$$
a=\frac{\iota_{\mu}[g]}{\iota_{\mu}\left[\chi_{\mu}\right]}=: \mathscr{A}_{\mu}[g]
$$

We combine the estimates of (2.15) in Proposition 3 on $\iota_{\mu}$ and (4.16) in Lemma 4 on $\iota_{\mu}\left[\chi_{\mu}\right]$ to prove the estimate for $\mathscr{A}_{\mu}$ in (4.19).

Now set

$$
\mathscr{P}_{\mu} g:=g-\frac{\iota_{\mu}[g]}{\iota_{\mu}\left[\chi_{\mu}\right]} \chi_{\mu}
$$

The previous estimate on $\iota_{\mu}\left[\chi_{\mu}\right]$ and the estimates on $\iota_{\mu}$ from in Proposition 3 and on $\chi_{\mu}$ from Lemma 3 cancel each other out to imply

$$
\left\|\mathscr{P}_{\mu}\right\|_{\mathbf{B}\left(O_{q}^{r}\right)} \leq C(q, r) .
$$

Next, substituting $a=\mathscr{A}_{\mu}[g]$ into (4.18) implies

$$
\mathscr{L}_{\mu} f=\mathscr{P}_{\mu} g .
$$

A direct calculation shows $\iota_{\mu}\left[\mathscr{P}_{\mu} g\right]=0$, so, by the solvability condition (2.16), $\mathscr{P}_{\mu} g$ is in the range of $\mathscr{L}_{\mu}$ in $O_{q}^{r}$. Using the notation of Proposition 2 for the inverse of $\mathscr{L}_{\mu}$ from its range to $O_{q}^{r+2}$, the equality (4.22) implies

$$
f=\mathscr{L}_{\mu}^{-1} \mathscr{P}_{\mu} g=: \mathscr{B}_{\mu} g .
$$

Then the coercive estimate (2.13) on $\mathscr{L}_{\mu}$ in Proposition 2 and (4.22) imply

$$
\left\|\mathscr{B}_{\mu} g\right\|_{r+j, q} \leq C(r, q) \mu^{-(j+1) / 2}\|g\|_{r, q} .
$$

Conversely, that $f, a$, and $g$ satisfy (4.18) if $a=\mathscr{A}_{\mu}[g]$ and $f=\mathscr{B}_{\mu} g$ follows from a straightforward algebraic rearrangement of the definitions of $\mathscr{A}_{\mu}$ and $\mathscr{B}_{\mu}$.

We return to our modified equation (4.10) for $\eta_{2}$ and $a$. We apply Proposition 4 with $f=\eta_{2}$ and $g=\sum_{k=1}^{6} \ell_{k}^{\mu}(\eta, a)$ to see that (4.10) is equivalent to the pair of fixed point equations

$$
\eta_{2}=\sum_{k=1}^{6} \mathscr{B}_{\mu} \ell_{k}^{\mu}(\boldsymbol{\eta}, a)=: \mathscr{N}_{2}^{\mu}(\boldsymbol{\eta}, a) \quad \text { and } \quad a=\sum_{k=1}^{6} \mathscr{A}_{\mu}\left[\ell_{k}^{\mu}(\boldsymbol{\eta}, a)\right]=: \mathscr{N}_{3}^{\mu}(\boldsymbol{\eta}, a) \text {. }
$$




\subsection{Construction of the Fixed Point Equation for $\eta_{1}$}

Last, we must construct an equation for $\eta_{1}$. The first equation in (4.3) reads

$$
\mathscr{H}_{c} \eta_{1}=\sum_{k=1}^{4} h_{k}^{\mu}(\boldsymbol{\eta}, a)-\kappa \delta \eta_{2} .
$$

By Proposition 1 we may invert $\mathscr{H}_{c}$, so that (4.24) is equivalent to

$$
\eta_{1}=\sum_{k=1}^{4} \mathscr{H}_{c}^{-1} h_{k}^{\mu}(\boldsymbol{\eta}, a)-\kappa \mathscr{H}_{c}^{-1} \delta \eta_{2}
$$

Next, we replace ${ }^{3} \eta_{2}$ with its fixed point equation $\eta_{2}=\mathscr{N}_{2}^{\mu}(\eta, a)$ and set

$$
h_{5}^{\mu}(\eta, a):=-\kappa \delta \mathscr{N}_{2}^{\mu}(\boldsymbol{\eta}, a) \quad \text { and } \quad \mathscr{N}_{1}^{\mu}(\boldsymbol{\eta}, a):=\sum_{k=1}^{5} \mathscr{H}_{c}^{-1} h_{k}^{\mu}(\boldsymbol{\eta}, a) .
$$

Then the three equations (4.23) and (4.25) are equivalent to the fixed point problem

$$
(\boldsymbol{\eta}, a)=\left(\mathscr{N}_{1}^{\mu}(\boldsymbol{\eta}, a), \mathscr{N}_{2}^{\mu}(\boldsymbol{\eta}, a), \mathscr{N}_{3}^{\mu}(\boldsymbol{\eta}, a)\right)=: \mathscr{N}_{\mu}(\boldsymbol{\eta}, a) .
$$

\subsection{Solution of the Nanopteron Fixed Point Problem (4.27)}

We will use the following fixed point lemma to solve our main problem; it appears as Theorem 4.10 in [29].

Lemma 5 Let $\mathscr{X}_{0}$ and $\mathscr{X}_{1}$ be reflexive Banach spaces with $\mathscr{X}_{1} \subseteq \mathscr{X}_{0}$. For $r>0$, let $\mathfrak{B}(r):=$ $\left\{x \in \mathscr{X}_{1} \mid\|x\|_{\mathscr{X}_{1}} \leq r\right\}$. Suppose that for some $r_{0}>0$, there is a map $\mathscr{N}: \mathfrak{B}\left(r_{0}\right) \rightarrow \mathscr{X}_{1}$ with the following properties.

(i) $\|x\|_{\mathscr{X}_{1}} \leq r_{0} \Longrightarrow\|\mathscr{N}(x)\|_{\mathscr{X}_{1}} \leq r_{0}$.

(ii) There exists $\alpha \in(0,1)$ such that

$$
\|x\|_{\mathscr{X}_{0}},\|\grave{x}\|_{\mathscr{X}_{1}} \leq r_{0} \Longrightarrow\|\mathscr{N}(x)-\mathscr{N}(\grave{x})\|_{\mathscr{X}_{0}} \leq \alpha\|x-\grave{x}\|_{\mathscr{X}_{0}} .
$$

Then there exists a unique $x_{\star} \in \mathscr{X}_{1}$ such that $\left\|x_{\star}\right\|_{\mathscr{X}_{1}} \leq r_{0}$ and $x_{\star}=\mathscr{N}\left(x_{\star}\right)$.

First we specify the Banach spaces (really, Hilbert spaces) in which we will work. With $q_{1}(c)$ and $q_{2}(c)$ from Proposition 1, fix $q_{\star}(c) \in\left(q_{1}(c), q_{2}(c)\right)$ and take $\bar{q}_{\star}(c) \in$ $\left(q_{1}(c), q_{\star}(c)\right)$. This restriction ensures that $\mathscr{H}_{c}$ is invertible from $E_{q_{\star}(c)}^{r+2} \rightarrow E_{q_{\star}(c), 0}^{r}$ and $E_{\bar{q}_{\star}(c)}^{r+2} \rightarrow E_{\bar{q}_{\star}(c), 0}^{r}$ for all $r \geq 0$. Now let

$$
\mathscr{X}_{r}:=\left\{\begin{array}{l}
E_{\bar{q}_{\star}(c)}^{3} \times O_{\bar{q}_{\star}(c)}^{3} \times \mathbb{R}, r=0 \\
E_{q_{\star}(c)}^{r+2} \times O_{q_{\star}(c)}^{r+2} \times \mathbb{R}, r \geq 1 .
\end{array}\right.
$$

\footnotetext{
${ }^{3}$ We could have made an additional change of variables in Sect. 2.1 that would have converted our symmetrized traveling wave problem (2.3) into one whose linear part is genuinely diagonal at $\mu=0$; Hoffman and Wright [25] do this in their small mass problem (see Section 1.3 of that paper). This would result in what we call $h_{5}^{\mu}$ in (4.26) being an $\mathscr{O}(\mu)$ term from the start, in which case replacing $\eta_{2}$ with $\mathscr{N}_{2}^{\mu}$ would be unnecessary. However, the new "almost diagonal" version of (2.3) and the resulting nanopteron system after Beale's ansatz would have a number of more complicated and opaque terms, and so we feel it is not worth the effort making this extra change of variables here.
} 
Next, for $r \geq 1$ and $\mu, \tau>0$, set

$$
\mathscr{U}_{\mu, \tau}^{r}:=\left\{(\boldsymbol{\eta}, a) \in \mathscr{X}^{r}\left|\|\eta\|_{r, q_{\star}(c)} \leq \tau \mu^{1 / 2},\right| a \mid \leq \tau \mu^{r / 2-1}\right\} .
$$

The following lemma, proved in Appendix D.1, is instrumental in our application of Lemma 5.

Lemma 6 There exist $\mu_{\star} \in\left(0, \min \left\{\mu_{\varsigma}, \mu_{\chi}\right\}\right)$ and $\tau_{\star}>0$ such that the following hold for all $\mu \in \mathrm{M}_{c, \kappa}:=\tilde{\mathrm{M}}_{c, \kappa} \cap\left(0, \mu_{\star}\right)$.

(i) If $(\boldsymbol{\eta}, a) \in \mathscr{U}_{\mu, \tau_{\star}}^{1}$, then $\mathscr{N}_{\mu}(\boldsymbol{\eta}, a) \in \mathscr{U}_{\mu, \tau_{\star}}^{1}$.

(ii) If $(\eta, a),(\grave{\eta}, \grave{a}) \in \mathscr{U}_{\mu, \tau_{\star}}^{1}$, then

$$
\left\|\mathscr{N}_{\mu}(\boldsymbol{\eta}, a)-\mathscr{N}_{\mu}(\grave{\eta}, \grave{a})\right\|_{\mathscr{X}_{0}} \leq \frac{1}{2}\|(\boldsymbol{\eta}, a)-(\grave{\eta}, \grave{a})\|_{\mathscr{X}_{0}} .
$$

(iii) For any $\tau>0$ and $r \geq 1$, if $(\eta, a) \in \mathscr{U}_{\mu, \tau_{\star}}^{1} \cap \mathscr{U}_{\mu, \tau}^{r}$, then there is $\tilde{\tau}>0$ such that $(\boldsymbol{\eta}, a) \in \mathscr{U}_{\mu, \widetilde{\tau}}^{r+1}$.

Fixing $r=3$, parts (i) and (ii) of Lemma 6 allow us to use Lemma 5 to find a unique $\left(\boldsymbol{\eta}_{\mu}, a_{\mu}\right) \in \mathscr{U}_{\mu, \tau_{\star}}^{1}$ such that $\left(\boldsymbol{\eta}_{\mu}, a_{\mu}\right)=\mathscr{N}_{\mu}\left(\boldsymbol{\eta}_{\mu}, a_{\mu}\right)$. Part (iii) then shows that $\boldsymbol{\eta}_{\mu}$ is smooth. Since $a_{\mu}=\mathscr{N}_{3}^{\mu}\left(\eta_{\mu}, a_{\mu}\right)$, we recall the definition of $\mathscr{N}_{3}^{\mu}$ in (4.23) and the estimates on the functional $\mathscr{A}_{\mu}$ from Proposition 4 to conclude that $a_{\mu}$ is small beyond all orders of $\mu$. We summarize these results formally in our main theorem below.

Theorem 3 Let $|c|>1, \kappa>0$, and $\mu \in \mathrm{M}_{c, \kappa} \cap\left(0, \mu_{\star}\right)$. Then there exists a unique $\left(\eta_{\mu}, a_{\mu}\right) \in$ $\mathscr{U}_{\mu, \tau_{\star}}^{1}$ such that $\left(\boldsymbol{\eta}_{\mu}, a_{\mu}\right)=\mathscr{N}_{\mu}\left(\eta_{\mu}, a_{\mu}\right)$. Moreover, $\boldsymbol{\eta}_{\mu} \in \cap_{r=0}^{\infty} E_{q_{\star}(c)}^{r} \times O_{q_{\star}(c)}$ and there are constants $C_{r}>0$ such that for each $r \geq 0$, we have

$$
\left\|\eta_{\mu}\right\|_{r, q_{\star}(c)} \leq C_{r} \mu \quad \text { and } \quad\left|a_{\mu}\right| \leq C_{r} \mu^{r} .
$$

\subsection{Additional Questions and Considerations}

The paper [12] also considers the "stiff internal spring" limit for MiM lattices. In this limit, one tries to solve the traveling wave problem for the lattice equations (1.1) in the limit as $\kappa \rightarrow \infty$. After defining new traveling wave profiles by rescaling those from (2.2) as $\theta_{1}=\rho_{1}$ and $\theta_{2}=\rho_{2} / \kappa$, the traveling wave problem for $\theta_{1}$ and $\theta_{2}$ becomes singularly perturbed like (2.3). Faver, Goodman, and Wright show that for a countable number of "antiresonance" values of $\kappa$ that diverge to $\infty$, the resulting system has solitary wave solutions. We expect that the techniques of this paper will carry over to show the existence of nanopteron traveling waves in the stiff internal spring limit for spring constants $\kappa$ bounded away from the antiresonance values. For that matter, a singularly perturbed stiff spring limit could be posed for the spring dimer FPUT lattice in [11], analogous to the small mass FPUT limit in [25].

The nanopteron estimates in (4.29) only establish that the amplitude $a_{\mu}$ of the ripple is small beyond all algebraic orders of $\mu$ and do not provide a lower bound on $a_{\mu}$, so that, conceivably, we could have $a_{\mu}=0$ for some $\mu$, and our nanopteron would really be a solitary wave. There are a number of numerical studies indicating that this may occur in diatomic FPUT lattices, see $[35,36,43,48]$. Ideally, we would also have an exponential upper bound of the form $\left|a_{\mu}\right| \leq e^{-C_{\star} / \mu}$ for some $C_{\star}=\mathscr{O}(1)$, which is what Sun and Shen achieve for the capillary-gravity water wave problem [46]. The same question can be posed for the amplitude of the ripples in the nanopteron papers [11,14,25,29].

Additionally, since we now know that solitary waves exist for the antiresonance mass ratios $\mu_{n}$ from (4.14), another natural question is the value of the $\operatorname{limit}_{\mu \rightarrow \mu_{n}} a_{\mu}$ for any 
fixed $n$. Does this limit equal zero? Of course, for this limit even to make sense, we must recall that we have found nanopterons with ripple amplitude $a_{\mu}$ where $\mu$ was restricted to the set $\mathrm{M}_{c, \kappa}$; as discussed at the start of Sect. 4.3, we could redefine $\mathrm{M}_{c, \kappa}$ to allow a more generous lower bound on $\mid \sin \left(\Omega_{\mu} / 2 \mid\right.$ and thus permit membership of a larger range of $\mu$ closer and closer to the $\mu_{n}$.

Resolution of any of these ripple questions will hinge on better knowledge of the oscillatory integral operator $\iota_{\mu}$ from (2.14), which is the critical operator in the definition (4.23) of our fixed point equation for $a$. One avenue toward this study would be the oscillatory integral theory developed by Lombardi [34] in analytic function spaces and invoked in a spatial dynamics approach to several FPUT lattice problems [26,27,49]. We are optimistic that techniques developed for further ripple analysis in any one of these heterogeneous lattice problems, FPUT or MiM, would readily translate to all the others due to the fundamentally similar structure of their traveling wave problems.

Last, we remark that the strictly linear bead-resonator force $r \mapsto \kappa r$ was very convenient for our construction and analysis of the traveling wave problem; for this reason, it is a popular force in the literature. In the antiresonance case, it facilitates in both [31] and [12] the reduction from two equations for two traveling wave profiles to only one equation, a key part of the analysis of each of these papers. It would therefore be interesting to add higher order terms to the bead-resonator force and see if the resulting complications are intrinsically challenging or merely technical.

Acknowledgements I thank Doug Wright for originally suggesting this problem to me and for his helpful comments and perspectives, as well as Atanas Stefanov for his interest and encouragement.

\section{Compliance with Ethical Standards}

Conflict of interest The authors declare that they have no conflict of interest.

Open Access This article is licensed under a Creative Commons Attribution 4.0 International License, which permits use, sharing, adaptation, distribution and reproduction in any medium or format, as long as you give appropriate credit to the original author(s) and the source, provide a link to the Creative Commons licence, and indicate if changes were made. The images or other third party material in this article are included in the article's Creative Commons licence, unless indicated otherwise in a credit line to the material. If material is not included in the article's Creative Commons licence and your intended use is not permitted by statutory regulation or exceeds the permitted use, you will need to obtain permission directly from the copyright holder. To view a copy of this licence, visit http://creativecommons.org/licenses/by/4.0/.

\section{A Fourier Analysis}

\section{A.1 Fourier Transforms and Series}

We use the following conventions for Fourier transforms and Fourier series. For $\mathbf{f} \in$ $L^{1}\left(\mathbb{R}, \mathbb{C}^{m}\right)$, we define

$$
\mathfrak{F}[\mathbf{f}](k)=\widehat{\mathbf{f}}(k):=\frac{1}{\sqrt{2 \pi}} \int_{-\infty}^{\infty} \mathbf{f}(x) e^{-i k x} d x
$$

and

$$
\mathfrak{F}^{-1}[\mathbf{f}](x)=\check{\mathbf{f}}(x):=\frac{1}{\sqrt{2 \pi}} \int_{-\infty}^{\infty} \mathbf{f}(k) e^{i k x} d x .
$$


If $\mathbf{f} \in L_{\text {per }}^{2}\left(\mathbb{R}, \mathbb{C}^{m}\right)$, we define

$$
\mathfrak{F}[\mathbf{f}](k)=\widehat{\mathbf{f}}(k)=\frac{1}{2 \pi} \int_{-\pi}^{\pi} \mathbf{f}(x) e^{-i k x} d x
$$

and we have

$$
\mathbf{f}(x)=\sum_{k=-\infty}^{\infty} \widehat{\mathbf{f}}(k) e^{i k x} .
$$

We regularly use the following identity governing the interaction of Fourier transforms and series with the shift operator $S^{d}$ :

$$
\widehat{S^{d} \mathbf{f}}(k)=e^{i k d} \widehat{\mathbf{f}}(k) .
$$

\section{A.2 Fourier Multipliers}

Suppose that $\widetilde{\mathscr{M}}: \mathbb{R} \rightarrow \mathbb{C}^{m \times m}$ is measurable and $r, s \in \mathbb{R}$ with

$$
\sup _{k \in \mathbb{R}} \frac{\|\widetilde{\mathscr{M}}(k)\|}{\left(1+k^{2}\right)^{(r-s) / 2}}<\infty .
$$

Then we may define the Fourier multiplier $\mathscr{M}: H^{r}\left(\mathbb{R}, \mathbb{C}^{m}\right) \rightarrow H^{s}\left(\mathbb{R}, \mathbb{C}^{m}\right)$ by

$$
\widehat{\mathscr{M}} \mathbf{f}(k)=\widetilde{\mathscr{M}}(k) \widehat{\mathbf{f}}(k) .
$$

The following is a slight generalization of Lemma 3 in [2], phrased in [13] as Lemma A.3. For $0 \leq q \leq \grave{q}$, we set

$$
S_{q, \grave{s}}:=\{z \in \mathbb{C}|q \leq| z \mid \leq \grave{q}\} .
$$

Lemma 7 (Beale) Let $0<q_{0} \leq q_{1}<q_{2}$ and suppose that $\widetilde{\mathscr{M}}: \mathbb{R} \rightarrow \mathbb{C}$ is a measurable function with the following properties.

( $\mathscr{M} \mathbf{1})$ The function $\widetilde{\mathscr{M}}$ is analytic on the strips $S_{0, q_{1}}$ and $S_{q_{1}, q_{2}}$.

( $\mathscr{M} 2)$ The function $\widetilde{\mathscr{M}}$ has finitely many zeros in $\mathbb{R}$, all of which are simple. Denote the collection of these zeros by $\mathfrak{P} \mathscr{M}$.

( $\mathscr{M} 3)$ There exist $C, z_{0}>0$ and $s \geq 0$ such that if $z \in S_{0, q_{1}} \cup S_{q_{1}, q_{2}}$ with $|z| \geq z_{0}$, then

$$
C|\operatorname{Re}(z)|^{s} \leq|\widetilde{\mathscr{M}}(z)| .
$$

Now let $\mathscr{M}$ be the Fourier multiplier with symbol $\widetilde{\mathscr{M}}$. There exist $q_{\star}, q_{\star \star}>0$ with $q_{1} \leq q_{\star}<q_{\star \star} \leq q_{2}$ such that if $q \in\left[q_{\star}, q_{\star \star}\right]$, then, for any $r \geq 0$, $\mathscr{M}$ is invertible from $H_{q}^{r+s}$ to the subspace

$$
\mathfrak{D}_{\mathscr{M}, q}^{r}:=\left\{f \in H_{q}^{r} \mid z \in \mathfrak{P} \mathscr{M} \Longrightarrow \widehat{f}(z)=0\right\}
$$

and, for $f \in \mathfrak{D}_{\mathscr{M}, q}^{r}$,

$$
\left\|\mathscr{M}^{-1} f\right\|_{r+s, q} \leq\left(\sup _{k \in \mathbb{R}} \frac{\left(1+k^{2}\right)^{s / 2}}{|\widetilde{\mathscr{M}}(k \pm i q)|}\right)\|f\|_{r, s} .
$$

In particular, if $q_{1}=q_{2}$, then the result above is true for all $0<q \leq q_{2}$. 


\section{B Construction of Periodic Solutions}

\section{B.1 Linear Analysis}

All of the information that we need for our periodic bifurcation argument is contained in the following proposition. We will need to recall the definitions and notation for the periodic Sobolev spaces introduced at the start of Sect. 3.

Proposition 5 The following statements are true when $\mu$ is sufficiently small.

(i) The eigenvalues $\lambda_{\mu}^{ \pm}$are real, even, bounded $2 \pi$-periodic functions. In particular,

$$
0 \leq \lambda_{\mu}^{-}(K) \leq \lambda_{\mu}^{-}(\pi)<\kappa(1+\mu)=\lambda_{\mu}^{+}(0)<\lambda_{\mu}^{+}(K) \leq \lambda_{\mu}^{+}(\pi) .
$$

(ii) $c^{2} \mu K^{2}-\lambda_{\mu}^{-}(K)=0$ if and only if $K=0$.

(iii) There exists a unique $\omega_{\mu}>0$ such that

$$
c^{2} \mu \omega_{\mu}^{2}-\lambda_{\mu}^{+}\left(\omega_{\mu}\right)=0
$$

Moreover,

$$
\Omega_{\mu}=\frac{\sqrt{\kappa(1+\mu)}}{c \sqrt{\mu}} \leq \omega_{\mu} \leq \frac{\sqrt{\lambda_{\mu}^{+}(\pi)}}{c \sqrt{\mu}}
$$

and

$$
\omega_{\mu}-\Omega_{\mu}=\mathscr{O}\left(\mu^{3 / 2}\right) .
$$

(iv) There exists $\alpha_{\mu} \in \mathbb{R}$ with $\alpha_{\mu}=\mathscr{O}(1)$ such that if

$$
v_{\mu}:=\sin \left(\frac{\omega_{\mu}}{2}\right) \alpha_{\mu}
$$

and

$$
\boldsymbol{v}_{\mu}(X):=\left(\begin{array}{c}
v_{\mu} \cos (X) \\
\sin (X)
\end{array}\right),
$$

then the kernel of $\Gamma_{\mu}\left[\omega_{\mu}\right]$ in $E_{\mathrm{per}, 0}^{r+2} \times O_{\mathrm{per}}^{r+2}, r \geq 0$, is spanned by $\boldsymbol{v}_{\mu}$.

(v) There exists $z_{\mu} \in \mathbb{R}$ with $z_{\mu}=\mathscr{O}\left(\mu^{2}\right)$ such that if

$$
\boldsymbol{v}_{\mu}^{*}(X):=\left(\begin{array}{c}
z_{\mu} \cos (X) \\
\sin (X)
\end{array}\right),
$$

then, given $\mathbf{f} \in E_{\mathrm{per}, 0}^{r+2} \times O_{\mathrm{per}}^{r+2}$ and $\mathbf{g} \in E_{\mathrm{per}, 0}^{r} \times O_{\mathrm{per}}^{r}$, we have $\Gamma_{\mu}\left[\omega_{\mu}\right] \mathbf{f}=\mathbf{g}$ if and only if $\left\langle\mathbf{g}, \boldsymbol{v}_{\mu}^{*}\right\rangle_{0}=0$.

(vi) There is a constant $C_{r}>0$, independent of $\mu$, such that if $\Gamma_{\mu}\left[\omega_{\mu}\right] \mathbf{f}=\mathbf{g}$ for $\mathbf{f} \in E_{\text {per, } 0}^{r+2} \times$ $O_{\text {per }}^{r+2}$ and $\mathbf{g} \in E_{\text {per }, 0}^{r} \times O_{\text {per }}^{r}$ with $\left\langle\mathbf{f}, v_{\mu}^{*}\right\rangle_{0}=\left\langle\mathbf{g}, v_{\mu}^{*}\right\rangle_{0}=0$, then $\|\mathbf{f}\|_{r+2} \leq C_{r}\|\mathbf{g}\|_{r}$.

Proof (i) From the definition of $P_{\mu}$ in (3.6), we see that $P_{\mu}(\cos (K))=\kappa^{2}+\mathscr{O}(\mu)$, so by taking $\mu$ sufficiently small we obtain $P_{\mu}(\cos (K))>0$, and therefore $\lambda_{ \pm}^{\mu}(K)$ are real for all $K$. That $\lambda_{\mu}^{ \pm}$are even, $2 \pi$-periodic, and continuously differentiable is then obvious. 
For the bounds, we first show that the critical points of $\lambda_{\mu}^{ \pm}$are precisely the set $\pi \mathbb{Z}$. We compute

$$
\left(\lambda_{\mu}^{ \pm}\right)^{\prime}(K)=\mu \sin (K)\left(1 \mp \frac{2 \mu \cos (K)+\kappa-(\kappa+2) \mu}{\sqrt{P_{\mu}(\cos (K))}}\right) .
$$

Next, we claim that when $\mu$ is sufficiently small, we have

$$
0<\frac{2 \mu \cos (K)+\kappa-(\kappa+2) \mu}{\sqrt{P_{\mu}(\cos (K))}}<1 .
$$

If this is true, then the only zeros of $\left(\lambda_{\mu}^{ \pm}\right)^{\prime}$ will be those of $\sin (\cdot)$. It is straightforward to check that if

$$
\mu<\min \left\{\frac{\kappa}{8}, \frac{\kappa}{4(\kappa+2)}\right\}
$$

then we have

$$
2 \mu \cos (K)+\kappa-(\kappa+2) \mu>0
$$

for all $K$. After some elementary algebraic rearrangements, we see that the inequality

$$
2 \mu \cos (K)+\kappa-(\kappa+2) \mu<\sqrt{P_{\mu}(\cos (K))}
$$

is equivalent to $4 \kappa^{2} \mu>0$, which is always true. And so (B.7) holds, and therefore the critical points of $\left(\lambda_{\mu}\right)^{ \pm}$are $\pi \mathbb{Z}$.

Corollary to (B.6) and (B.7) are the useful inequalities

$$
\left|\left(\lambda_{\mu}^{+}\right)^{\prime}(K)\right| \leq \mu|K| \quad \text { and } \quad\left|\left(\lambda_{\mu}^{-}\right)^{\prime}(K)\right| \leq 2 \mu|K|,
$$

which we will need in later parts of the proof.

Now we are able to bound $\lambda_{\mu}^{ \pm}$. We refer to (B.6) to see that $\left(\lambda_{\mu}^{ \pm}\right)^{\prime}(K)=0$ if and only if $\sin (K)=0$, which happens if and only if $K \in \pi \mathbb{Z}$. Since $\lambda_{\mu}^{ \pm}$are $2 \pi$-periodic, we only need to evaluate these functions at $K=0$ and $K=\pi$. We compute

$$
\lambda_{\mu}^{-}(0)=\frac{\kappa(1+\mu)}{2}-\frac{\sqrt{P_{\mu}(1)}}{2}=\frac{\kappa(1+\mu)}{2}-\frac{\sqrt{\kappa^{2}(1+\mu)^{2}}}{2}=0
$$

and

$$
\lambda_{\mu}^{-}(\pi)=\frac{\kappa(1+\mu)}{2}+2 \mu-\frac{\sqrt{P_{\mu}(-1)}}{2} .
$$

The quantity $P_{\mu}(-1)$ will also be important for understanding $\lambda_{\mu}^{+}(\pi)$, so we provide the estimates

$$
\frac{\sqrt{P_{\mu}(-1)}}{2}<\frac{\kappa(1+\mu)}{2}+2 \mu \text { and } \frac{\kappa(1+\mu)}{2}<2 \mu+\frac{\sqrt{P_{\mu}(-1)}}{2},
$$

which are proved with a few easy rearrangements of some equivalent inequalities. We conclude

$$
\frac{\kappa(1+\mu)}{2}+2 \mu-\frac{\sqrt{P_{\mu}(-1)}}{2}>0,
$$

and so

$$
0 \leq \lambda_{\mu}^{-}(K) \leq \lambda_{\mu}^{-}(\pi)<\kappa(1+\mu)
$$


for all $K$.

Next, we evaluate

$$
\lambda_{\mu}^{+}(0)=\kappa(1+\mu) \quad \text { and } \quad \lambda_{\mu}^{+}(\pi)=\frac{\kappa(1+\mu)}{2}+2 \mu+\frac{\sqrt{P_{\mu}(-1)}}{2} .
$$

By (B.9), we have

$$
\frac{\kappa(1+\mu)}{2}+2 \mu+\frac{\sqrt{P_{\mu}(-1)}}{2}>\frac{\kappa(1+\mu)}{2}+\frac{\kappa(1+\mu)}{2}=\kappa(1+\mu)=\lambda_{\mu}^{+}(0),
$$

and so

$$
\lambda_{\mu}^{+}(0)=\kappa(1+\mu) \leq \lambda_{\mu}^{+}(K) \leq \lambda_{\mu}^{+}(\pi)
$$

for all $K$.

(ii) We use the second inequality in (B.8) and the calculation $\lambda_{\mu}^{-}(0)=0$ to estimate

$$
\begin{aligned}
c^{2} \mu K^{2}-\lambda_{\mu}^{-}(K) & =\int_{0}^{K}\left(2 c^{2} \mu s-\left(\lambda_{\mu}^{-}\right)^{\prime}(s)\right) d s \geq \int_{0}^{K}\left(2 c^{2} \mu s-2 \mu s\right) d s \\
& =\int_{0}^{K} 2 \mu\left(c^{2}-1\right) s d s=\mu\left(c^{2}-1\right) K^{2} .
\end{aligned}
$$

Since $c^{2}>1$, we have

$$
c^{2} K^{2}-\lambda_{\mu}^{-}(K)>0
$$

for all $K>0$, and so by evenness $c^{2} K^{2}-\lambda_{\mu}^{-}(K) \neq 0$ for all $K \neq 0$.

(iii) Since

$$
\kappa(1+\mu) \leq \lambda_{\mu}^{+}(K) \leq \lambda_{\mu}^{+}(\pi)
$$

for all $K$, we see that if $0<K<\sqrt{\kappa(1+\mu) / c^{2} \mu}=\Omega_{\mu}$, then

$$
c^{2} \mu K^{2}-\lambda_{\mu}^{+}(K)<0,
$$

while if $K>\sqrt{\lambda_{\mu}^{+}(\pi) / c^{2} \mu}$, then

$$
c^{2} \mu K^{2}-\lambda_{\mu}^{+}(K)>0 .
$$

Consequently, there exists at least one $\omega_{\mu}>0$ with

$$
\Omega_{\mu} \leq \omega_{\mu} \leq \sqrt{\frac{\lambda_{\mu}^{+}(\pi)}{c^{2} \mu}}
$$

such that

$$
c^{2} \mu \omega_{\mu}^{2}-\lambda_{\mu}^{+}\left(\omega_{\mu}\right)=0 .
$$

For the uniqueness of $\omega_{\mu}$, we use the first inequality in (B.8) to bound

$$
\frac{d}{d K}\left[c^{2} \mu K^{2}-\lambda_{\mu}^{+}(K)\right]=2 c^{2} \mu K-\left(\lambda_{\mu}^{+}\right)^{\prime}(K) \geq\left(2 c^{2}-1\right) \mu K>0
$$

when $K>0$, so $c^{2} \mu K^{2}-\lambda_{\mu}^{+}(K)$ is strictly increasing on $(0, \infty)$ and therefore has at most one positive root. By evenness this root is unique on all of $\mathbb{R}$. 
Last, we prove the estimate (B.2). We expand the square root in the definition of $\lambda_{\mu}^{+}$ with Taylor's theorem to calculate

$$
\begin{aligned}
0 & =c^{2} \mu \omega_{\mu}^{2}-\lambda_{\mu}^{+}\left(\omega_{\mu}\right)=c^{2} \mu \omega_{\mu}^{2}-\frac{\kappa(1+\mu)}{2}+\frac{\left(2 \cos \left(\omega_{\mu}\right)-2\right) \mu}{2} \\
& -\frac{1}{2}\left(\sqrt{\kappa^{2}}+\frac{1}{2 \sqrt{\kappa^{2}}}(2 \kappa)\left(2 \cos \left(\omega_{\mu}\right)-2+\kappa\right) \mu+\mathscr{O}\left(\mu^{2}\right)\right)=c^{2} \mu \omega_{\mu}^{2}-\kappa(1+\mu)+\mathscr{O}\left(\mu^{2}\right) .
\end{aligned}
$$

Then, recalling that

$$
c^{2} \mu \Omega_{\mu}-\kappa(1+\mu)=0,
$$

we subtract this equation from (B.10) to find

$$
c^{2} \mu\left(\omega_{\mu}+\Omega_{\mu}\right)\left(\omega_{\mu}-\Omega_{\mu}\right)=\mathscr{O}\left(\mu^{2}\right) .
$$

Since $\omega_{\mu}$ and $\Omega_{\mu}$ are both $\mathscr{O}\left(\mu^{-1 / 2}\right)$, we conclude that

$$
\omega_{\mu}-\Omega_{\mu}=\mathscr{O}\left(\mu^{3 / 2}\right) .
$$

(iv) If $\Gamma_{\mu}\left[\omega_{\mu}\right] \boldsymbol{\phi}=0$ and $\widehat{\boldsymbol{\phi}}(k) \neq 0$, then either

$$
c^{2} \mu\left(\omega_{\mu} k\right)^{2}=\lambda_{\mu}^{-}\left(\omega_{\mu} k\right) \quad \text { or } \quad c^{2} \mu\left(\omega_{\mu} k\right)^{2}=\lambda_{\mu}^{+}\left(\omega_{\mu} k\right) .
$$

By part (ii), the first case holds if and only if $k=0$. But we have assumed that $\phi_{1}$ is mean-zero and $\phi_{2}$ is odd, which forces $\widehat{\boldsymbol{\phi}}(0)=0$, so only the second equality in (B.11) is possible.

In that case, part (iii) implies $\omega_{\mu} k= \pm \omega_{\mu}$, so $k= \pm 1$. We conclude that if $\Gamma_{\mu}\left[\omega_{\mu}\right] \phi=0$ and $\widehat{\phi}(k) \neq 0$, then $k= \pm 1$, so

$$
\phi(X)=e^{-i X} \widehat{\boldsymbol{\phi}}(-1)+e^{i X} \widehat{\boldsymbol{\phi}}(1),
$$

where $\widehat{\boldsymbol{\phi}}(1)$ is an eigenvector of $\widetilde{\mathscr{D}}_{\mu}\left[\omega_{\mu}\right]$ corresponding to the eigenvalue $\lambda_{\mu}^{+}\left(\omega_{\mu}\right)$. Since $\phi_{1}$ is even and $\phi_{2}$ is odd, (B.12) rearranges into

$$
\phi(X)=2\left(\begin{array}{c}
\widehat{\phi}_{1}(1) \cos (X) \\
i \widehat{\phi}_{2}(1) \sin (X)
\end{array}\right)
$$

The definition of $\widetilde{\mathscr{D}}_{\mu}$ in (3.4) and this eigenvalue-eigenvector relationship imply

$$
\widehat{\boldsymbol{\phi}}(1)=a\left(\begin{array}{c}
i \alpha_{\mu} \sin \left(\frac{\omega_{\mu}}{2}\right) \\
1
\end{array}\right), \quad \alpha_{\mu}:=\frac{2 \kappa}{\mu\left(2 \cos \left(\omega_{\mu}\right)-2\right)+\lambda_{\mu}^{+}\left(\omega_{\mu}\right)}
$$

for some scalar $a$. Note that the definition of $\lambda_{\mu}^{+}$in (3.5) gives

$$
\left(2 \cos \left(\omega_{\mu}\right)-2\right) \mu+\lambda_{\mu}^{ \pm}\left(\omega_{\mu}\right)=\frac{\kappa}{2}+\frac{\sqrt{P_{\mu}\left(\cos \left(\omega_{\mu}\right)\right)}}{2}+\mathscr{O}(\mu),
$$

and so for $\mu$ sufficiently small the denominator of $\alpha_{\mu}$ is bounded away from zero. That is, $\alpha_{\mu}=\mathscr{O}(1)$, and combining (B.13) and (B.3) shows that $\boldsymbol{\phi}=a \boldsymbol{v}_{\mu}$ for some scalar $a$, where $\boldsymbol{v}_{\mu}$ is defined as in (B.4).

(v) It suffices to show that the kernel of $\Gamma_{\mu}\left[\omega_{\mu}\right]^{*}$ is spanned by a vector $v_{\mu}^{*}$ of the form given in (B.5). Here $\Gamma_{\mu}\left[\omega_{\mu}\right]^{*}: E_{\mathrm{per}, 0}^{r} \times O_{\mathrm{per}}^{r} \rightarrow E_{\mathrm{per}, 0}^{r+2} \times O_{\mathrm{per}}^{r+2}$ is the adjoint of $\Gamma_{\mu}\left[\omega_{\mu}\right]$. 
Then part (v) will follow by classical functional analysis.

Elementary theory of Fourier multipliers tells us that this adjoint satisfies

$$
\mathfrak{F}\left[\Gamma_{\mu}\left[\omega_{\mu}\right]^{*} \phi\right](k)=\frac{1}{\left(1+k^{2}\right)^{2}}\left(-c^{2} \mu \omega_{\mu}^{2} k^{2}+I^{\mu} \widetilde{\mathscr{D}}_{\mu}\left[\omega_{\mu} k\right]^{*}\right) \widehat{\phi}(k),
$$

where $\widetilde{\mathscr{D}}_{\mu}[K]^{*}$ is the conjugate transpose of the matrix $\widetilde{\mathscr{D}}_{\mu}[K]$. Now let $\boldsymbol{\phi} \in E_{\text {per, } 0}^{r} \times$ $O_{\text {per }}^{r}$ be a nontrivial element of the kernel of $\Gamma_{\mu}\left[\omega_{\mu}\right]^{*}$. Then $c^{2} \mu \omega_{\mu} k^{2}$ is an eigenvalue of $I^{\mu} \widetilde{\mathscr{D}}_{\mu}\left[\omega_{\mu} k\right]^{*}$ corresponding to the eigenvector $\widehat{\boldsymbol{\phi}}(k)$. Since $I^{\mu} \widetilde{\mathscr{D}}_{\mu}\left[\omega_{\mu} k\right]^{*}=$ $\left(\widetilde{\mathscr{D}}_{\mu}\left[\omega_{\mu} k\right] I^{\mu}\right)^{*}$, and the eigenvalues of $\widetilde{\mathscr{D}}_{\mu}\left[\omega_{\mu} k\right] I^{\mu}$ are the real-valued functions $\lambda_{\mu}^{ \pm}\left(\omega_{\mu} k\right)$, the same critical alternative (B.11) must hold. Repeating the analysis from part (iv), we obtain (B.13) again, so we calculate $\widehat{\phi}(1)$. This time we find

$$
\widehat{\boldsymbol{\phi}}(1)=a\left(\begin{array}{c}
z_{\mu} \\
1
\end{array}\right), \quad z_{\mu}:=\frac{2 \mu^{2}}{\mu\left(2 \cos \left(\omega_{\mu}\right)-2\right)+\lambda_{\mu}^{+}\left(\omega_{\mu}\right)} \sin \left(\frac{\omega_{\mu}}{2}\right),
$$

so $z_{\mu}=\mathscr{O}\left(\mu^{2}\right)$.

(vi) If $\Gamma_{\mu}\left[\omega_{\mu}\right] \mathbf{f}=\mathbf{g}$ for some $\mathbf{f} \in E_{\mathrm{per}, 0}^{r+2} \times O_{\text {per }}^{r+2}$ and $\mathbf{g} \in E_{\mathrm{per}, 0}^{r} \times O_{\mathrm{per}}^{r}$, then, for $k \neq 0$, we have

$$
\left(\mathbb{1}-\frac{1}{c^{2} \mu \omega_{\mu}^{2} k^{2}} \widetilde{\mathscr{D}}_{\mu}\left[\omega_{\mu} k\right] I^{\mu}\right) \widehat{\mathbf{f}}(k)=-\frac{1}{c^{2} \mu \omega_{\mu}^{2} k^{2}} \widehat{\mathbf{g}}(k) .
$$

Here $\mathbb{1}$ is the $2 \times 2$ identity matrix. For $|k| \geq 2$, we will show that the $\infty$-norm of the matrix

$$
\frac{1}{c^{2} \omega_{\mu}^{2} k^{2}} \widetilde{\mathscr{D}}_{\mu}\left[\omega_{\mu} k\right] I^{\mu}
$$

is smaller than 1 uniformly in $\mu$ and $k$. This, along with estimating $\mu \omega_{\mu}^{2}=\mathscr{O}(1)$ on the right side of (B.15), enables us to invert the matrix on the left side and obtain an estimate of the form $|\widehat{\mathbf{f}}(k)| \leq C|\widehat{\mathbf{g}}(k)| / k^{2}$ for $|k| \geq 2$. When $|k|=1$, we will prove directly that $|\widehat{\mathbf{f}}( \pm 1)| \leq C|\widehat{\mathbf{f}}( \pm 1)|$. Combining these estimates, Plancherel's theorem will imply the desired inequality $\|\mathbf{f}\|_{r+2} \leq C_{r}\|\mathbf{g}\|_{r}$.

Referring to the definition of $\widetilde{\mathscr{D}}_{\mu}$ in (3.4), we see that by taking $\mu$ sufficiently small, we have

$$
\left\|\widetilde{\mathscr{D}}_{\mu}\left[\omega_{\mu} k\right] I^{\mu}\right\|_{\infty} \leq 2 \kappa
$$

By the estimates on $\omega_{\mu}$ from (B.1), this implies

$$
\frac{1}{c^{2} \mu \omega_{\mu}^{2} k^{2}}\left\|\widetilde{\mathscr{D}}_{\mu}\left[\omega_{\mu} k\right] I^{\mu}\right\|_{\infty} \leq \frac{2}{k^{2}}<\frac{1}{2}
$$

when $|k| \geq 2$. A Neumann series argument allows us to conclude

$$
\left\|\left(\mathbb{1}-\frac{1}{c^{2} \mu \omega_{\mu}^{2} k^{2}} \widetilde{\mathscr{D}}_{\mu}\left[\omega_{\mu} k\right] I^{\mu}\right)^{-1}\right\|_{\infty} \leq 2
$$

Now we consider the case $|k|=1$. In this case, the equality $\widehat{\Gamma_{\mu}\left[\omega_{\mu}\right]} \mathbf{f}(1) \cdot \mathbf{e}_{1}=\widehat{\mathbf{g}}(1) \cdot \mathbf{e}_{1}$ is equivalent to 


$$
-c^{2} \mu \omega_{\mu}^{2} \widehat{f}_{1}(1)-\mu\left(2 \cos \left(\omega_{\mu}\right)-2\right) \widehat{f}_{1}(1)+2 i \kappa \sin \left(\frac{\omega_{\mu}}{2}\right) \widehat{f}_{2}(1)=\widehat{g}_{1}(1) .
$$

It is here that we need the additional hypothesis from part (vi) that

$$
\left\langle\mathbf{f}, \boldsymbol{v}_{\mu}^{*}\right\rangle_{0}=\left\langle\mathbf{g}, \boldsymbol{v}_{\mu}^{*}\right\rangle_{0}=0 .
$$

Since $f_{1}$ and $g_{1}$ are even and $f_{2}$ and $g_{2}$ are odd, these equalities and the definition of $v_{\mu}^{*}$ in (B.5) imply

$$
\widehat{f_{2}}(1)=-i z_{\mu} \widehat{f_{1}}(1) \quad \text { and } \quad \widehat{g}_{2}(1)=-i z_{\mu} \widehat{g}_{1}(1) .
$$

We can therefore remove $\widehat{f_{2}}(1)$ from the left side of (B.16) and rewrite that equation as

$$
\left(-c^{2} \mu \omega_{\mu}^{2}-\mu\left(2 \cos \left(\omega_{\mu}\right)-2\right)+2 z_{\mu} \kappa \sin \left(\frac{\omega_{\mu}}{2}\right)\right) \widehat{f}_{1}(1)=\widehat{g}_{1}(1) .
$$

Since $\mu \omega_{\mu}^{2}=\mathscr{O}(1)$ and $z_{\mu}=\mathscr{O}\left(\mu^{2}\right)$, the prefactor on $\widehat{f_{1}}(1)$ is bounded away from zero uniformly in $\mu$ when $\mu$ is small. We conclude $\left|\widehat{f}_{1}(1)\right| \leq C\left|\widehat{g}_{1}(1)\right|$.

If $z_{\mu}=0$, then $\left|\widehat{f}_{2}(1)\right|=0=\left|\widehat{g}_{2}(1)\right|$, and there is nothing to prove for the estimate on the second component. Otherwise, we can write

$$
\frac{1}{\left|z_{\mu}\right|}\left|\widehat{f}_{2}(1)\right|=\left|\widehat{f}_{1}(1)\right| \leq C\left|\widehat{g}_{1}(1)\right|=\frac{C}{\left|z_{\mu}\right|}\left|\widehat{g}_{2}(1)\right|,
$$

and thus $\left|\widehat{f}_{2}(1)\right| \leq C\left|\widehat{g}_{2}(1)\right|$.

The estimates for $k=-1$ now follow by the even-odd symmetry of $\mathbf{f}$ and $\mathbf{g}$.

\section{B.2 The Bifurcation Argument}

Proposition 5 is analogous to Lemma B.1 in [25], which contained all the information necessary for their construction of the periodic traveling waves. Since the argument for the MiM periodic solutions would proceed exactly as the one for the small mass FPUT periodics, we do not feel the need to give it here and sketch it only fleetingly; a longer summary can be found in Appendix C of [13], and the full argument takes place in Appendix B of [25]. This argument bears some strong similarities with the periodic proofs for the long wave problem in Appendix C of [14] and relies on a number of Lipschitz estimates for scaled Fourier multipliers, which are detailed in [14] and also Appendix D of [10].

Here, then, in brief is the argument. One wishes to solve $\boldsymbol{\Phi}_{\mu}(\boldsymbol{\phi}, \mu)=0$ from (3.2) and makes the revised ansatz

$$
\boldsymbol{\phi}=a \boldsymbol{v}_{c}+a \boldsymbol{\psi} \quad \text { and } \quad \omega=\omega_{\mu}+\xi,
$$

where $\psi \in E_{\text {per }, 0}^{2} \times O_{\text {per }}^{2}$ and $\xi \in \mathbb{R}$. This is similar to the ansatz made in the actual proof of the bifurcation from simple eigenvalues theorem by Crandall and Rabinowitz [7]. Then one uses the various properties delineated in Proposition 5 to rearrange the problem $\boldsymbol{\Phi}_{\mu}\left(a \boldsymbol{v}_{c}+a \boldsymbol{\psi}, \omega_{\mu}+\xi\right)$ into a fixed point problem for $\boldsymbol{\psi}$ and $\xi$, where $a$ and $\mu$ are parameters. Specifically, one finds an equation for $\xi$ by projecting onto the one-dimensional span of $\boldsymbol{v}_{c}$ and then an equation for $\psi$ by projecting onto the orthogonal complement of this span; parts (v) and (vi) of Proposition 5 are critical here. A quantitative fixed point argument, using many of the Fourier multiplier estimates discussed above, finishes the job. 


\section{Proofs for the Construction of the Nanopteron Fixed Point Argument}

\section{C.1 Proof of Proposition 2}

This proof is similar to the proofs of Lemmas A.8 and A.9 in [14]. However, the notation and estimates are sufficiently different that we feel it worthwhile to spell out the details here.

Fix $|c|>1, \kappa>0$, and $\mu \in(0,1)$, and let

$$
\widetilde{\mathscr{M}}(z):=c^{2} z^{2}-\kappa(1+\mu)
$$

so that

$$
\widehat{\mathscr{L}_{\mu} f}(k)=\widetilde{\mathscr{M}}(\sqrt{\mu} k) \widehat{f}(k) .
$$

Since $\widetilde{\mathscr{M}}$ is just a quadratic polynomial with the distinct roots $\pm \sqrt{\kappa(1+\mu)} /|c|$, it is straightforward to see that all the hypotheses of Lemma 7 are satisfied. In particular, they hold for any $q>0$, since the only zeros of $\widetilde{\mathscr{M}}$ are real.

Lemma 7 also gives the estimate

$$
\left\|\mathscr{L}_{\mu}^{-1}\right\|_{\mathbf{B}\left(\mathfrak{D}_{\mu, q}^{r}, O_{q}^{r+j}\right)} \leq \sup _{k \in \mathbb{R}} \frac{\left(1+k^{2}\right)^{j / 2}}{|\widetilde{\mathscr{M}}(\sqrt{\mu}(k+i q))|} .
$$

We estimate this supremum further:

$$
\begin{aligned}
& \sup _{k \in \mathbb{R}} \frac{\left(1+k^{2}\right)^{j / 2}}{|\widetilde{\mathscr{M}}(\sqrt{\mu}(k+i q))|}=\sup _{k \in \mathbb{R}} \frac{\left(1+(k / \sqrt{\mu})^{2}\right)^{j / 2}}{|\widetilde{\mathscr{M}}(k+i \sqrt{\mu} q)|}=\sup _{k \in \mathbb{R}}\left(\frac{\left(1+(k / \sqrt{\mu})^{2}\right)^{j / 2}}{\left(1+k^{2}\right)^{j / 2}}\right)\left(\frac{\left(1+k^{2}\right)^{j / 2}}{\widetilde{\mathscr{M}}(k+i \sqrt{\mu} q)}\right) \\
& \leq\left(\sup _{k \in \mathbb{R}} \frac{\left(1+(k / \sqrt{\mu})^{2}\right)^{j / 2}}{\left(1+k^{2}\right)^{j / 2}}\right)\left(\sup _{k \in \mathbb{R}} \frac{\left(1+k^{2}\right)^{j / 2}}{\widetilde{\mathscr{M}}(k+i \sqrt{\mu} q)}\right) .
\end{aligned}
$$

Some elementary algebraic rearrangements imply

$$
\sup _{k \in \mathbb{R}} \frac{\left(1+(k / \sqrt{\mu})^{2}\right)^{j / 2}}{\left(1+k^{2}\right)^{j / 2}} \leq \frac{1}{\mu^{j / 2}} .
$$

We claim more generally that if $0<|\tau|<\tau_{0}$ for some $\tau_{0}>0$, then there is a constant $C>0$, independent of $\tau$ (but dependent on $c, \kappa$, and $\tau_{0}$ ) such that

$$
\sup _{k \in \mathbb{R}} \frac{\left(1+k^{2}\right)^{j / 2}}{\widetilde{\mathscr{M}}(k+i \tau)} \leq \frac{C}{|\tau|}
$$

for $j=0,1,2$. From this our desired estimate (2.13) follows.

So, we just need to prove this claim (C.1). First, if $z \in \mathbb{C}$ with $|z| \geq 2 \sqrt{\kappa} /|c|$, then the reverse triangle inequality implies

$$
|\widetilde{\mathscr{M}}(z)| \geq \frac{c^{2}|z|^{2}}{2},
$$

from which we see that if $k \in \mathbb{R}$ with $|k| \geq 2 \sqrt{\kappa} /|c|$ and $0<|\tau|<\tau_{0}$, then

$$
\frac{|\widetilde{\mathscr{M}}(k+i \tau)|}{1+k^{2}} \geq \frac{c^{2}|k+i \tau|^{2}}{2\left(1+k^{2}\right)} \geq \frac{c^{2} k^{2}}{2\left(1+k^{2}\right)} \geq \frac{c^{2}}{4}=\left(\frac{c^{2}}{4 \tau_{0}}\right) \tau_{0} \geq\left(\frac{c^{2}}{4 \tau_{0}}\right)|\tau| .
$$


Then for $j=0,1,2$,

$$
\sup _{\substack{k \in \mathbb{R} \\|k| \geq 2 \sqrt{\kappa} /|c|}} \frac{\left(1+k^{2}\right)^{j} / 2}{|\widetilde{\mathscr{M}}(k+i \tau)|} \leq \sup _{\substack{k \in \mathbb{R} \\|k| \geq 2 \sqrt{\kappa} /|c|}} \frac{1+k^{2}}{|\widetilde{\mathscr{M}}(k+i \tau)|} \leq\left(\frac{4 \tau_{0}}{c^{2}}\right) \frac{1}{|\tau|} .
$$

When $|k| \leq 2 \sqrt{\kappa} /|c|$, we use a partial fractions decomposition to rewrite

$$
\frac{1}{\widetilde{\mathscr{M}}(z)}=-\frac{1}{2 \sqrt{\kappa(1+\mu)}(c z+\sqrt{\kappa(1+\mu)})}+\frac{1}{2 \sqrt{\kappa(1+\mu)}(c z-\sqrt{\kappa(1+\mu)})},
$$

and then it is apparent that

$$
\frac{1}{|c(k+i \tau) \pm \sqrt{\kappa(1+\mu)}|}=\frac{1}{\sqrt{(c k \pm \sqrt{\kappa(1+\mu)})^{2}+c^{2} \tau^{2}}} \leq \frac{1}{|c \tau|} .
$$

Thus

$$
\sup _{\substack{k \in \mathbb{R} \\|k| \leq 2 \sqrt{\kappa} /|c|}} \frac{1+k^{2}}{|\widetilde{\mathscr{M}}(k+i \tau)|} \leq\left(\frac{1+4 \kappa / c^{2}}{|c|}\right) \frac{1}{|\tau|} .
$$

This completes the proof of (C.1) and also of Proposition 2.

\section{C.2 Proof of the Identity (4.13)}

With $\chi_{\mu}$ defined in (4.9) and $\iota_{\mu}$ in (2.14), we calculate

$$
\begin{gathered}
\iota_{\mu}\left[\chi_{\mu}\right]=-2 \mu^{2} v_{\mu} \int_{-\infty}^{\infty}\left(\delta\left[\sigma_{c} \cos \left(\Omega_{\mu} \cdot\right)\right]\right)(x) \sin \left(\Omega_{\mu} x\right) d x \\
=2 \mu^{2} v_{\mu} \int_{-\infty}^{\infty} \sigma_{c}(x) \cos \left(\Omega_{\mu} x\right)\left(\delta\left[\sin \left(\Omega_{\mu} \cdot\right)\right]\right)(x) \\
d x=4 \mu^{2} v_{\mu} \sin \left(\frac{\Omega_{\mu}}{2}\right) \int_{-\infty}^{\infty} \sigma_{c}(x) \cos ^{2}\left(\Omega_{\mu} x\right) d x .
\end{gathered}
$$

To obtain the second equality, we used the skew-adjointness of $\delta$ in the $L^{2}$-inner product, and for the third we directly calculated $\delta\left[\sin \left(\Omega_{\mu} \cdot\right)\right]$ using the addition formulas for sine.

Next, we have

$$
\sigma_{c}(x) \cos ^{2}\left(\omega_{\mu} x\right)=\sigma_{c}(x)\left(\frac{e^{i \omega_{\mu} x}+e^{-i \omega_{\mu} x}}{2}\right)^{2}=\frac{\sigma_{c}(x)}{4}+\frac{\sigma_{c}(x)}{4}\left(e^{2 i \omega_{\mu} x}+e^{-2 i \omega_{\mu} x}\right) .
$$

A variation on the Riemann-Lebesgue lemma (such as that given in Lemma A.4 of [14], which also proves Proposition 3 in this paper) shows

$$
\int_{-\infty}^{\infty} \frac{\sigma_{c}(x)}{4}\left(e^{2 i \omega_{\mu} x}+e^{-2 i \omega_{\mu} x}\right) d x=\mathscr{O}\left(\mu^{\infty}\right) .
$$

Since $\sigma_{c}(x)>0$ for all $x$, we conclude Thus

$$
\iota_{\mu}\left[\chi_{\mu}\right]=\mu^{2} v_{\mu} \sin \left(\frac{\omega_{\mu}}{2}\right)\left\|\sigma_{c}\right\|_{L^{1}}+\mathscr{O}\left(\mu^{\infty}\right) .
$$

Now, recall from (3.9) that

$$
v_{\mu}=\sin \left(\frac{\omega_{\mu}}{2}\right) \alpha_{\mu},
$$


where $\alpha_{\mu}=\mathscr{O}(1)$. Also, part (iii) of Lemma 2 tells us

$$
\omega_{\mu}-\Omega_{\mu}=\mathscr{O}\left(\mu^{3 / 2}\right) .
$$

It follows that

$$
\iota_{\mu}\left[\chi_{\mu}\right]=\mu^{2} \sin ^{2}\left(\frac{\Omega_{\mu}}{2}\right) \alpha_{\mu}\left\|\sigma_{c}\right\|_{L^{1}}+\mathscr{O}\left(\mu^{7 / 2}\right)
$$

\section{The Proof of Theorem 3}

For simplicity in this appendix, we abbreviate $q_{\star}:=q_{\star}(c)$ and $\bar{q}_{\star}(c):=\bar{q}_{\star}$. These decay rate constants were selected in Sect. 4.6.

\section{D.1 Proof of Lemma 6}

We rely on the following lemma, whose proof is in Appendix D.2.

Lemma 8 For all $r \geq 3$, there is $C_{\star}(r)>0$ such that the following estimates hold for any $\mu \in \mathbf{M}_{c, \kappa} \cap\left(0, \mu_{\chi}\right)$.

(i) If $\eta \in E_{q_{\star}}^{3} \times O_{q_{\star}}^{3}$ and $|a| \leq a_{\mathrm{per}}$, then

$$
\left\|\mathscr{N}_{\mu}(\boldsymbol{\eta}, a)\right\|_{\mathscr{X}_{1}} \leq C_{\star}(3)\left(\mu^{1 / 2}+\mu^{1 / 2}|a|+\mu^{1 / 2}\|\boldsymbol{\eta}\|_{3, q_{\star}}+|a|^{2}+\|\boldsymbol{\eta}\|_{3, q_{\star}}^{2}\right) .
$$

(ii) If $\eta, \grave{\eta} \in E_{q_{\star}}^{3} \times O_{q_{\star}}^{3}$ and $|a|,|\grave{a}| \leq a_{\mathrm{per}}$, then

$$
\begin{aligned}
& \left\|\mathscr{N}_{\mu}(\boldsymbol{\eta}, a)-\mathscr{N}_{\mu}(\grave{\boldsymbol{\eta}}, \grave{a})\right\|_{\mathscr{X}_{0}} \\
& \quad \leq C_{\star}(3)\left(\mu^{1 / 2}+\|\boldsymbol{\eta}\|_{3, q_{\star}}+\|\grave{\eta}\|_{3, q_{\star}}+|a|+|\grave{a}|\right)\left(\|\boldsymbol{\eta}-\grave{\eta}\|_{3, \bar{q}_{\star}}+|a-\grave{a}|\right) .
\end{aligned}
$$

(iii) If $\eta \in E_{q_{\star}}^{r} \times O_{q_{\star}}^{r}$ and $|a| \leq a_{\mathrm{per}}$, then

$$
\begin{aligned}
& \left\|\mathscr{N}_{1}^{\mu}(\boldsymbol{\eta}, a)\right\|_{r+1, q_{\star}}+\left\|\mathscr{N}_{2}^{\mu}(\boldsymbol{\eta}, a)\right\|_{r+1, q_{\star}} \\
& \quad \leq C_{\star}(r)\left(\mu^{1 / 2}+\mu^{3 / 2-r / 2}|a|+\|\boldsymbol{\eta}\|_{r, q_{\star}}+\mu^{1-r / 2}|a|\|\boldsymbol{\eta}\|_{r, q_{\star}}+\mu^{1-r / 2}|a|^{2}+\|\boldsymbol{\eta}\|_{r, q_{\star}}^{2}\right)
\end{aligned}
$$

and

$$
\left|\mathscr{N}_{3}^{\mu}(\boldsymbol{\eta}, a)\right| \leq C_{\star}(r)\left(\mu^{r / 2}+\mu^{r / 2-1}\|\boldsymbol{\eta}\|_{r, q_{\star}}+\mu^{r / 2-1}\|\boldsymbol{\eta}\|_{r, q_{\star}}^{2}+|a|\|\boldsymbol{\eta}\|_{r, q_{\star}}+|a|^{2}\right) .
$$

Now we prove Lemma 6 . Abbreviate $C_{\star}=C_{\star}(3)$ from the lemma above and set

$$
\mu_{\star}=\min \left\{\frac{1}{2}, \mu_{\varsigma}, \mu_{\chi}, \frac{a_{\mathrm{per}}^{2}}{4 C_{\star}^{2}}, \frac{1}{64 C_{\star}^{2}\left(1+2 C_{\star}\right)^{2}}, \frac{1}{4 C_{\star}^{2}\left(1+8 C_{\star}\right)^{2}}\right\} .
$$

Let $\tau_{\star}=2 C_{\star}$. Observe that if $(\eta, a) \in \mathscr{U}_{\mu, \tau_{\star}}^{1}$, where this set was defined in (4.28), and $0<\mu<\mu_{\star}$, then

$$
|a| \leq \tau_{\star} \mu^{3 / 2-1}=2 C_{\star} \mu^{1 / 2} \leq a_{\mathrm{per}}
$$


so $\mathscr{N}_{\mu}(\boldsymbol{\eta}, a)$ is indeed defined. In the following, fix $0<\mu<\mu_{\star}$.

To prove part (i), fix $(\eta, a) \in \mathscr{U}_{\mu, \tau_{\star}}^{1}$ and use (D.1) to estinate

$$
\begin{aligned}
& \left\|\mathscr{N}_{1}^{\mu}(\boldsymbol{\eta}, a)\right\|_{3, q_{\star}}+\left\|\mathscr{N}_{2}^{\mu}(\boldsymbol{\eta}, a)\right\|_{3, q_{\star}}+\left|\mathscr{N}_{3}^{\mu}(\boldsymbol{\eta}, a)\right| \\
& \quad \leq C_{\star}\left(\mu^{1 / 2}+\mu^{1 / 2}|a|+\mu^{1 / 2}\|\boldsymbol{\eta}\|_{3, q_{\star}}+|a|^{2}+\|\boldsymbol{\eta}\|_{3, q_{\star}}^{2}\right) \leq C_{\star} \mu^{1 / 2}\left(1+4 C_{\star} \mu^{1 / 2}+8 C_{\star}^{2} \mu^{1 / 2}\right) \\
& =C_{\star} \mu^{1 / 2}\left(1+\mu^{1 / 2}\left(4 C_{\star}\right)\left(1+2 C_{\star}\right)\right)<\frac{3}{2} C_{\star} \mu^{1 / 2}<\tau_{\star} \mu^{1 / 2} .
\end{aligned}
$$

Consequently, $\mathscr{N}_{\mu}(\eta, a) \in \mathscr{U}_{\mu, \tau_{\star}}^{1}$ as well.

To prove part (ii), take $(\boldsymbol{\eta}, a),(\grave{\eta}, \grave{a}) \in \mathscr{U}_{\mu, \tau_{\star}}^{1}$ and estimate

$$
C_{\star}\left(\mu^{1 / 2}+\|\eta\|_{3, q_{\star}}+\|\grave{\eta}\|_{q_{\star}}+|a|+|\grave{a}|\right) \leq C_{\star}\left(\mu^{1 / 2}+4 \tau_{\star} \mu^{1 / 2}\right)=C_{\star} \mu^{1 / 2}\left(1+8 C_{\star}\right)<\frac{1}{2} .
$$

Then part (ii) follows from (D.2).

Last, for part (iii), assume $(\boldsymbol{\eta}, a) \in \mathscr{U}_{\mu, \tau_{\star}}^{1} \cap \mathscr{U}_{\mu, \tau}^{r}$. Since $(\boldsymbol{\eta}, a) \in \mathscr{U}_{\mu, \tau_{\star}}^{1}, \mathscr{N}_{\mu}(\boldsymbol{\eta}, a)$ is defined. Now we use (D.3) to bound $\left\|\mathscr{N}_{1}^{\mu}(\boldsymbol{\eta}, a)\right\|_{r+1, q_{\star}}+\left\|\mathscr{N}_{2}^{\mu}(\boldsymbol{\eta}, a)\right\|_{r+1, q_{\star}}$ above by

$$
\begin{gathered}
C_{\star}(r)\left(\mu^{1 / 2}+\mu^{3 / 2-r / 2}|a|+\|\boldsymbol{\eta}\|_{r, q}+\mu^{1-r / 2}|a|\|\boldsymbol{\eta}\|_{r, q}+\mu^{1-r / 2}|a|^{2}+\|\boldsymbol{\eta}\|_{r, q}^{2}\right) \\
\leq C_{\star}(r)\left(\mu^{1 / 2}+3 \tau \mu^{1 / 2}+2 \tau^{2} \mu^{1 / 2}+\tau^{2} \mu^{r / 2-1}\right) \leq C_{\star}(r)\left(3 \tau+2 \tau^{2}\right) \mu^{1 / 2} .
\end{gathered}
$$

Note that for $r \geq 3$, we have $r / 2-1 \geq 1 / 2$, so we are indeed able to factor out a power of $\mu^{1 / 2}$ in the last inequality above.

Next, we want to show

$$
\left|\mathscr{N}_{3}^{\mu}(\eta, a)\right| \leq \tilde{\tau} \mu^{(r+1) / 2-1}=\tilde{\tau} \mu^{r / 2-1 / 2}
$$

for some $\tilde{\tau}>0$. We use (D.4) to estimate

$$
\begin{aligned}
\left|\mathscr{N}_{3}^{\mu}(\boldsymbol{\eta}, a)\right| & \leq C_{\star}(r)\left(\mu^{r / 2}+\mu^{r / 2-1}\|\boldsymbol{\eta}\|_{r, q}+\mu^{r / 2-1}\|\boldsymbol{\eta}\|_{r, q}^{2}+|a|\|\boldsymbol{\eta}\|_{r, q}+|a|^{2}\right) \\
& \leq C_{\star}(r)\left(\mu^{r / 2}+2 \tau \mu^{r / 2-1 / 2}+\tau^{2} \mu^{r / 2}+\tau^{2} \mu^{r-2}\right) \\
& \leq C_{\star}(r) \mu^{r / 2-1 / 2}\left(\mu^{1 / 2}+2 \tau+\tau^{2} \mu^{1 / 2}+\tau^{2} \mu^{r / 2-3 / 2}\right) .
\end{aligned}
$$

We set

$$
\tilde{\tau}:=\max \left\{C_{\star}(r)\left(3 \tau+2 \tau^{2}\right), C_{\star}\left(\mu_{\star}^{1 / 2}+2 \tau+\tau^{2} \mu_{\star}^{1 / 2}+\tau^{2} \mu_{\star}^{r / 2-3 / 2}\right\} .\right.
$$

This concludes the proof of Lemma 6 .

\section{D.2 Proof of Lemma 8}

\section{D.2.1 Proof of Part (i)}

Set

$$
\mathscr{R}_{\text {map }}^{\mu}(\boldsymbol{\eta}, a):=\mu^{1 / 2}+\mu^{1 / 2}|a|+\mu^{1 / 2}\|\boldsymbol{\eta}\|_{3, q_{\star}}+|a|^{2}+\|\boldsymbol{\eta}\|_{3, q_{\star}}^{2} .
$$

We will show that

$$
\left\|\mathscr{N}_{1}^{\mu}(\boldsymbol{\eta}, a)\right\|_{3, q_{\star}}+\left\|\mathscr{N}_{2}^{\mu}(\boldsymbol{\eta}, a)\right\|_{3, q_{\star}}+\left|\mathscr{N}_{3}^{\mu}(\boldsymbol{\eta}, a)\right| \leq C \mathscr{R}_{\text {map }}^{\mu}(\boldsymbol{\eta}, a),
$$

which implies (D.1). 
We first claim that

$$
\left\|h_{k}^{\mu}(\boldsymbol{\eta}, a)\right\|_{r, q_{\star}} \leq C(r) \mathfrak{H}_{\text {map }, r}^{\mu}(\boldsymbol{\eta}, a), k=1, \ldots, 4,
$$

and

$$
\left\|\ell_{k}^{\mu}(\eta, a)\right\|_{r, q_{\star}} \leq C(r) \mathfrak{L}_{\text {map }, r}^{\mu}(\eta, a), k=1, \ldots, 6,
$$

where

$$
\mathfrak{H}_{\text {map }, r}^{\mu}(\boldsymbol{\eta}, a):=\mu^{1-r / 2}|a|+\mu\|\boldsymbol{\eta}\|_{r, q_{\star}}+\mu^{1-r / 2}|a|\|\boldsymbol{\eta}\|_{r, q_{\star}}+\|\boldsymbol{\eta}\|_{r, q_{\star}}^{2}
$$

and

$$
\mathfrak{L}_{\text {map }, r}^{\mu}(\boldsymbol{\eta}, a):=\mu^{2}+\mu^{3-r / 2}|a|+\mu\|\boldsymbol{\eta}\|_{r, q_{\star}}+\mu\|\boldsymbol{\eta}\|_{r, q_{\star}}^{2}+\mu^{2-r / 2}|a|\|\boldsymbol{\eta}\|_{r, q_{\star}}+\mu^{2-r / 2}|a|^{2} .
$$

We prove these estimates in Appendix 1.

Then from the definition of $\mathscr{N}_{2}^{\mu}$ in (4.23) and the estimates on $\mathscr{B}_{\mu}$ in Proposition 4 with $j=0$, we have

$$
\begin{aligned}
\left\|\mathscr{N}_{2}^{\mu}(\boldsymbol{\eta}, a)\right\|_{3, q_{\star}} & \leq \sum_{k=1}^{6}\left\|\mathscr{B}_{\mu} \ell_{k}^{\mu}(\boldsymbol{\eta}, a)\right\|_{3, q_{\star}} \leq C \mu^{-1 / 2} \sum_{k=1}^{6}\left\|\ell_{k}^{\mu}(\boldsymbol{\eta}, a)\right\|_{3, q_{\star}} \\
& \leq C \mu^{-1 / 2} \mathfrak{L}_{\text {map }, 3}^{\mu}(\boldsymbol{\eta}, a) \leq C \mathscr{R}_{\text {map }}^{\mu}(\boldsymbol{\eta}, a) .
\end{aligned}
$$

Next, we use the definition of $\mathscr{N}_{1}^{\mu}$ in (4.26) and the invertibility of $\mathscr{H}_{c}$ from Proposition 1 to estimate $\left\|\mathscr{N}_{2}^{\mu}(\boldsymbol{\eta}, a)\right\|_{3, q_{\star}} \leq \sum_{k=1}^{5}\left\|\mathscr{H}_{c}^{-1} h_{k}^{\mu}(\boldsymbol{\eta}, a)\right\|_{3, q_{\star}} \leq C \sum_{k=1}^{4}\left\|h_{k}^{\mu}(\boldsymbol{\eta}, a)\right\|_{1, q_{\star}}+C\left\|\mathscr{N}_{2}^{\mu}(\boldsymbol{\eta}, a)\right\|_{3, q_{\star}}$.

We have

$$
\left\|h_{k}^{\mu}(\boldsymbol{\eta}, a)\right\|_{1, q_{\star}} \leq C \mathfrak{H}_{\text {map }, 1}^{\mu}(\boldsymbol{\eta}, a) \leq C \mathscr{R}_{\text {map }}^{\mu}(\boldsymbol{\eta}, a),
$$

and so, with the previous estimates on $\mathscr{N}_{2}^{\mu}$, we find

$$
\left\|\mathscr{N}_{1}^{\mu}(\boldsymbol{\eta}, a)\right\|_{3, q_{\star}} \leq C \mathscr{R}_{\text {map }}^{\mu}(\eta, a) .
$$

Last, we use the definition of $\mathscr{N}_{3}^{\mu}$ from (4.23) and the estimates on $\mathscr{A}_{\mu}$ from Proposition 4 to produce the general estimate

$$
\left|\mathscr{N}_{3}^{\mu}(\boldsymbol{\eta}, a)\right| \leq \sum_{k=1}^{6}\left|\mathscr{A}_{\mu}\left[\ell_{k}^{\mu}(\boldsymbol{\eta}, a)\right]\right| \leq C(r) \mu^{r / 2-2} \sum_{k=1}^{6}\left\|\ell_{k}^{\mu}(\boldsymbol{\eta}, a)\right\|_{r, q_{\star}} \leq C(r) \mu^{r / 2-2} \mathfrak{L}_{\text {map }, r}^{\mu}(\boldsymbol{\eta}, a) .
$$

Taking $r=3$, we have

$$
\left|\mathscr{N}_{3}^{\mu}(\boldsymbol{\eta}, a)\right| \leq C \mu^{-1 / 2} \mathfrak{L}_{\mathrm{map}, 3}^{\mu}(\boldsymbol{\eta}, a),
$$

and so, by (D.10),

$$
\left|\mathscr{N}_{3}^{\mu}(\boldsymbol{\eta}, a)\right| \leq C \mathscr{R}_{\text {map }}^{\mu}(\eta, a) .
$$

Combining (D.10), (D.11), and (D.13) gives (D.5). 


\section{D.2.2 Proof of Part (ii)}

Set

$$
\mathscr{R}_{\mathrm{lip}}^{\mu}(\boldsymbol{\eta}, a):=\mu^{1 / 2}+\|\boldsymbol{\eta}\|_{3, \bar{q}_{\star}}+\|\grave{\eta}\|_{3, \bar{q}_{\star}}+|a|+|\grave{a}|
$$

We will show that

$$
\begin{aligned}
& \left\|\mathscr{N}_{1}^{\mu}(\boldsymbol{\eta}, a)-\mathscr{N}_{1}^{\mu}(\grave{\eta}, \grave{a})\right\|_{3, \bar{q}_{\star}}+\left\|\mathscr{N}_{2}^{\mu}(\boldsymbol{\eta}, a)-\mathscr{N}_{2}^{\mu}(\grave{\boldsymbol{\eta}}, \grave{a})\right\|_{3, \bar{q}_{\star}}+\left|\mathscr{N}_{3}^{\mu}(\boldsymbol{\eta}, a)-\mathscr{N}_{3}^{\mu}(\grave{\boldsymbol{\eta}}, \grave{a})\right| \\
& \quad \leq C \mathscr{R}_{\text {lip }}^{\mu}(\boldsymbol{\eta}, a)\left(\|\boldsymbol{\eta}-\grave{\boldsymbol{\eta}}\|_{3, q_{\star}}+|a-\grave{a}|\right),
\end{aligned}
$$

which will imply (D.2).

To do so, we will exploit a mismatch of regularity and work with $r=1$ for the $h_{k}^{\mu}$ terms but $r=3$ for the $\ell_{k}^{\mu}$ terms. Namely, we claim that

$$
\left\|h_{k}^{\mu}(\boldsymbol{\eta}, a)-h_{k}^{\mu}(\grave{\eta}, \grave{a})\right\|_{1, \bar{q}_{\star}} \leq C \mathfrak{H}_{\text {lip }}^{\mu}(\boldsymbol{\eta}, \grave{\boldsymbol{\eta}}, a, \grave{a})\left(\|\boldsymbol{\eta}-\grave{\boldsymbol{\eta}}\|_{1, \bar{q}_{\star}}+|a-\grave{a}|\right), \quad k=1, \ldots, 4,
$$

and

$$
\left\|\ell_{k}^{\mu}(\boldsymbol{\eta}, a)-\ell_{k}^{\mu}(\grave{\eta}, \grave{a})\right\|_{3, \bar{q}_{\star}} \leq C \mathfrak{L}_{\text {lip }}^{\mu}(\boldsymbol{\eta}, \grave{\eta}, a, \grave{a})\left(\|\boldsymbol{\eta}-\grave{\eta}\|_{3, \bar{q}_{\star}}+|a-\grave{a}|\right), \quad k=1, \ldots, 6,
$$

where

$$
\mathfrak{H}_{\text {lip }}^{\mu}(\boldsymbol{\eta}, \grave{\eta}, a, \grave{a}):=\mu^{1 / 2}+\|\boldsymbol{\eta}\|_{1, q_{\star}}+\|\grave{\boldsymbol{\eta}}\|_{1, q_{\star}}+|a|+|\grave{a}|,
$$

while

$$
\mathfrak{L}_{\text {lip }}^{\mu}(\boldsymbol{\eta}, \grave{\boldsymbol{\eta}}, a, \grave{a}):=\mu+\mu^{1 / 2}\left(\|\boldsymbol{\eta}\|_{3, q_{\star}}+\|\grave{\boldsymbol{\eta}}\|_{3, q_{\star}}+|a|+|\grave{a}|\right) .
$$

We prove these estimates in Appendix 1, too.

Assuming this to be true, we use the definition of $\mathscr{N}_{2}^{\mu}$ in (4.23) and the estimates on $\mathscr{B}_{\mu}$ from Proposition 4 to estimate.

$$
\begin{aligned}
& \left\|\mathscr{N}_{2}^{\mu}(\boldsymbol{\eta}, a)-\mathscr{N}_{2}^{\mu}(\grave{\boldsymbol{\eta}}, \grave{a})\right\|_{3, \bar{q}_{\star}} \leq \sum_{k=1}^{6}\left\|\mathscr{B}_{\mu}\left(\ell_{k}^{\mu}(\boldsymbol{\eta}, a)-\ell_{k}^{\mu}(\grave{\boldsymbol{\eta}}, \grave{a})\right)\right\|_{3, \bar{q}_{\star}} \\
& \quad \leq C \mu^{-1 / 2} \sum_{k=1}^{6}\left\|\ell_{k}^{\mu}(\boldsymbol{\eta}, a)-\ell_{k}^{\mu}(\grave{\boldsymbol{\eta}}, \grave{a})\right\|_{3, \bar{q}_{\star}} \leq C \mu^{-1 / 2} \mathfrak{L}_{\text {lip }}^{\mu}(\boldsymbol{\eta}, \grave{\boldsymbol{\eta}}, a, \grave{a}) \leq C \mathscr{R}_{\text {lip }}^{\mu}(\boldsymbol{\eta}, \grave{\boldsymbol{\eta}}, a, \grave{a}) .
\end{aligned}
$$

Next, we use the definition of $\mathscr{N}_{1}^{\mu}$ from (4.26) and the smoothing properties of $\mathscr{H}_{C}$ from Proposition 1 to bound

$$
\begin{aligned}
& \left\|\mathscr{N}_{1}^{\mu}(\boldsymbol{\eta}, a)-\mathscr{N}_{1}^{\mu}(\grave{\boldsymbol{\eta}}, \grave{a})\right\|_{3, \bar{q}_{\star}} \leq \sum_{k=1}^{5}\left\|\mathscr{H}_{c}^{-1}\left(h_{k}^{\mu}(\boldsymbol{\eta}, a)-h_{k}^{\mu}(\grave{\boldsymbol{\eta}}, \grave{a})\right)\right\|_{3, \bar{q}_{\star}} \\
& \quad \leq C \sum_{k=1}^{4}\left\|h_{k}^{\mu}(\boldsymbol{\eta}, a)-h_{k}^{\mu}(\grave{\boldsymbol{\eta}}, \grave{a})\right\|_{1, \bar{q}_{\star}}+C\left\|\mathscr{N}_{2}^{\mu}(\boldsymbol{\eta}, a)-\mathscr{N}_{2}^{\mu}(\grave{\boldsymbol{\eta}}, \grave{a})\right\|_{3, q_{\star}} \\
& \quad \leq C \mathfrak{H}_{\text {lip }}^{\mu}(\boldsymbol{\eta}, \grave{\boldsymbol{\eta}}, a, \grave{a})+C \mathscr{R}_{\text {lip }}^{\mu}(\boldsymbol{\eta}, \grave{\boldsymbol{\eta}}, a, \grave{a}) .
\end{aligned}
$$

It is clear that $\mathfrak{H}_{\text {lip }}^{\mu}(\boldsymbol{\eta}, \grave{\eta}, a, \grave{a}) \leq \mathscr{R}_{\text {lip }}^{\mu}(\boldsymbol{\eta}, \grave{\eta}, a, \grave{a})$, and so we have

$$
\left\|\mathscr{N}_{1}^{\mu}(\boldsymbol{\eta}, a)-\mathscr{N}_{1}^{\mu}(\grave{\eta}, \grave{a})\right\|_{3, \bar{q}_{\star}} \leq C \mathscr{R}_{\text {lip }}^{\mu}(\boldsymbol{\eta}, \grave{\boldsymbol{\eta}}, a, \grave{a}) .
$$


Last, we use the definition of $\mathscr{N}_{3}^{\mu}$ from (4.23) and the estimates on $\mathscr{A}_{\mu}$ from Proposition 4 to find

$$
\left|\mathscr{N}_{3}^{\mu}(\boldsymbol{\eta}, a)-\mathscr{N}_{3}^{\mu}(\grave{\boldsymbol{\eta}}, \grave{a})\right| \leq \sum_{k=1}^{6}\left|\mathscr{A}_{\mu}\left[\ell_{k}^{\mu}(\boldsymbol{\eta}, a)-\ell_{k}^{\mu}(\grave{\boldsymbol{\eta}}, \grave{a})\right]\right| \leq C \mu^{-1 / 2} \sum_{k=1}^{6}\left\|\ell_{k}^{\mu}(\boldsymbol{\eta}, a)-\ell_{k}^{\mu}(\grave{\boldsymbol{\eta}}, \grave{a})\right\|_{3, \bar{q}_{\star}},
$$

and, recognizing this last term from (D.15), we conclude that

$$
\left|\mathscr{N}_{3}^{\mu}(\boldsymbol{\eta}, a)-\mathscr{N}_{3}^{\mu}(\grave{\eta}, \grave{a})\right| \leq C \mathscr{R}_{\text {lip }}^{\mu}(\boldsymbol{\eta}, \grave{\eta}, a, \grave{a})
$$

as well. Combining (D.15), (D.16), and (D.17), we establish (D.14).

\section{D.2.3 Proof of Part (iii)}

Let

$\mathscr{R}_{\text {boot }, r}^{\mu}(\boldsymbol{\eta}, a):=\mu^{1 / 2}+\mu^{3 / 2-r / 2}|a|+\|\boldsymbol{\eta}\|_{r, q_{\star}}+\mu^{1-r / 2}|a|\|\boldsymbol{\eta}\|_{r, q_{\star}}+\mu^{1-r / 2}|a|^{2}+\|\boldsymbol{\eta}\|_{r, q_{\star}}^{2}$.

We show that

$$
\left\|\mathscr{N}_{1}^{\mu}(\eta, a)\right\|_{r+1, q_{\star}}+\left\|\mathscr{N}_{2}^{\mu}(\eta, a)\right\|_{r+1, q_{\star}} \leq C(r) \mathscr{R}_{\mathrm{boot}, r}^{\mu}(\boldsymbol{\eta}, a),
$$

which will imply (D.3).

First, using the definition of $\mathscr{N}_{2}^{\mu}$ in (4.23) and the estimates on $\mathscr{B}_{\mu}$ with $j=1$ from Proposition 4, we have

$$
\left\|\mathscr{N}_{2}^{\mu}(\boldsymbol{\eta}, a)\right\|_{r+1, q_{\star}} \leq \sum_{k=1}^{6}\left\|\mathscr{B}_{\mu} \ell_{k}^{\mu}(\boldsymbol{\eta}, a)\right\|_{r+1, q_{\star}} \leq C(r) \mu^{-1} \sum_{k=1}^{6}\left\|\ell_{k}^{\mu}(\eta, a)\right\|_{r, q_{\star}} .
$$

This, in conjunction with the mapping bounds (D.7) and (D.9), gives

$$
\left\|\mathscr{N}_{2}^{\mu}(\eta, a)\right\|_{r+1, q_{\star}} \leq C(r) \mu^{-1} \mathfrak{L}_{\text {map }, r}^{\mu}(\eta, a) \leq C(r) \mathscr{R}_{\text {boot }, r}^{\mu}(\eta, a) .
$$

Next, the definition of $\mathscr{N}_{1}^{\mu}$ in (4.26) and the smoothing properties of $\mathscr{H}_{c}$ imply

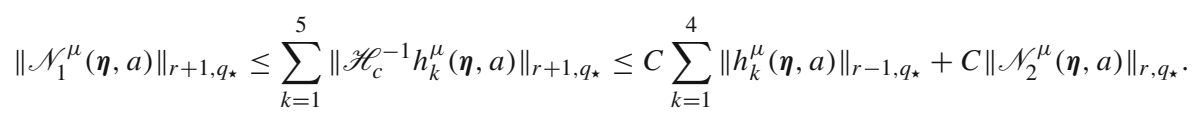

The mapping bounds (D.6) and (D.6) provide

$$
\left\|h_{k}^{\mu}(\boldsymbol{\eta}, a)\right\|_{r-1, q_{\star}} \leq C(r)\left(\mu^{1-(r-1) / 2}|a|+\mu\|\eta\|_{r-1, q_{\star}}+\mu^{1-(r-1) / 2}|a|\|\eta\|_{r-1, q_{\star}}+\|\eta\|_{r-1, q_{\star}}^{2}\right) .
$$

Since $1-(r-1) / 2=3 / 2-r / 2$, we may further bound

$$
\left\|h_{k}^{\mu}(\eta, a)\right\|_{r-1, q_{\star}} \leq C(r) \mathscr{R}_{\text {boot }, r}^{\mu}(\eta, a),
$$

and thus

$$
\left.\left\|\mathscr{N}_{1}^{\mu}(\eta, a)\right\|_{r+1, q_{\star}} \leq C(r)\right) \mathscr{R}_{\mathrm{boot}, r}^{\mu}(\eta, a) .
$$

By (D.12), we have 


$$
\begin{aligned}
& \left|\mathscr{N}_{3}^{\mu}(\boldsymbol{\eta}, a)\right| \leq C(r) \mu^{r / 2-2} \mathfrak{L}_{\text {map }, r}^{\mu}(\boldsymbol{\eta}, a) \\
& \quad=C(r)\left(\mu^{r / 2}+\mu^{3}|a|+\mu^{r / 2-1}\|\boldsymbol{\eta}\|_{r, q_{\star}}+\mu^{r / 2-1}\|\boldsymbol{\eta}\|_{r, q_{\star}}^{2}+|a|\|\boldsymbol{\eta}\|_{r, q_{\star}}+|a|^{2}\right) .
\end{aligned}
$$

This proves (D.4) and completes the proof of Lemma 8.

Remark 2 Observe from (D.9) that the first term in $\mathfrak{L}_{\text {map }, r}^{\mu}$ is $\mu^{2}$. This is an artifact of the refined leading order limit of Theorem 1 and appears in the estimate on $\ell_{1}^{\mu}$ below in Appendix 1. Had we not made the refined leading order limit, this term $\ell_{1}^{\mu}$ would be instead $-\mathscr{G}_{c}\left(\sigma_{c}, \mu\right) \cdot \mathbf{e}_{2}$; see the calculations at the start of Sect. 4.1 and in particular (4.4). As we discussed in Sect. 2.4, we have only $\left\|\mathscr{G}_{c}\left(\sigma_{c}, \mu\right) \cdot \mathbf{e}_{2}\right\|_{r, q_{c}}=\mathscr{O}(\mu)$. But then in taking the estimate (D.19), multiplying by $\mu^{-1}$ would leave us with an $\mathscr{O}(1)$ term in $\mathscr{R}_{\text {boot }, r}^{\mu}(\boldsymbol{\eta}, a)$, and this would ruin the proof of part (iii) of Lemma 6 given in Appendix 1.

\section{D.3 Estimates on the Terms $h_{1}^{\mu}, \ldots, h_{5}^{\mu}$ and $\ell_{1}^{\mu}, \ldots, \ell_{6}^{\mu}$}

We recall that the terms $h_{1}^{\mu}, \ldots, h_{4}^{\mu}$ and $\ell_{1}^{\mu}, \ldots, \ell_{3}^{\mu}$ were defined in (4.4), while $\ell_{4}^{\mu}$ and $\ell_{5}^{\mu}$ appeared in (4.7), $\ell_{6}^{\mu}$ in (4.8), and $h_{5}^{\mu}$ in (4.26).

We will rely heavily on the following collection of abstract estimates, which was stated as Proposition G.1 in [13]. Its proof is a combination of calculus and techniques from [14], in particular Lemma A.2 in that paper.

Proposition 6 (i) If $f \in H_{q}^{r}$ and $g \in W^{r, \infty}$, then

$$
\|f g\|_{r, q} \leq\|f\|_{r, q}\|g\|_{W^{r, \infty}} .
$$

(ii) If $f, g \in H_{q}^{r}$, then

$$
\|f g\|_{r, q} \leq\|\operatorname{sech}(q \cdot)\|_{W^{r, \infty}}\|f\|_{r, q}\|g\|_{r, q} .
$$

(iii) If $f \in W^{r, \infty}$ and $\omega \in \mathbb{R}$, then

$$
\|f(\omega \cdot)\|_{W^{r, \infty}} \leq\left(\max _{0 \leq k \leq r}|\omega|^{k}\right)\|f\|_{W^{r, \infty}} .
$$

(iv) If $f \in H_{q_{2}}^{r} ; g \in W^{r, \infty} ; \omega, \grave{\omega} \in \mathbb{R}$; and $0<q_{1}<q_{2}$, then

$$
\|f \cdot(g(\omega \cdot)-g(\grave{\omega} \cdot))\|_{r, q_{1}} \leq C\left(r, q_{2}-q_{1}\right)\left(\max _{0 \leq k \leq r} \operatorname{Lip}\left(\partial_{x}^{k}[g]\right)\right)\|f\|_{r, q_{2}}\|g\|_{W^{r, \infty}}|\omega-\grave{\omega}|,
$$

where, for a function $h: \mathbb{R} \rightarrow \mathbb{C}$, the Lipschitz constant $\operatorname{Lip}(h)$ is

$$
\operatorname{Lip}(h):=\sup _{\substack{x, \grave{x} \in \mathbb{R} \\ x \neq \grave{x}}}\left|\frac{h(x)-h(\grave{x})}{x-\grave{x}}\right| .
$$

(v) If $f \in H_{q_{2}}^{r} ; g$, ’̀ $\in W^{r, \infty} ; \omega, \grave{\omega} \in \mathbb{R}$; and $0<q_{1}<q_{2}$, then

$$
\begin{aligned}
& \|f \cdot(g(\omega \cdot)-\grave{g}(\grave{\omega} \cdot))\|_{r, q_{1}} \\
& \leq C\left(r, q_{2}-q_{1}\right)\|f\|_{r, q_{2}}\left[\left(\max _{0 \leq k \leq r} \operatorname{Lip}\left(\partial_{x}^{k}[g]\right)\right)\|g\|_{W^{r, \infty}}|\omega-\grave{\omega}|+\left(\max _{0 \leq k \leq r}|\grave{\omega}|^{k}\right)\|g-\grave{g}\|_{W^{r, \infty}}\right] .
\end{aligned}
$$


Using Theorem 2, specifically the Lipschitz estimates in (3.10), and the scaling estimate in (D.24), it is straightforward to deduce the following additional estimates on the periodic profiles.

Lemma 9 (i) Let $\mu \in\left(0, \mu_{\text {per }}\right)$. Then

$$
\sup _{|a| \leq a_{\mathrm{per}}}\left|\omega_{\mu}^{a}\right|+\left\|\phi_{\mu, 1}^{a}\right\|_{W^{r, \infty}} \leq C(r) \mu^{1-r / 2} .
$$

(ii) Let $\mu \in\left(0, \mu_{\mathrm{per}}\right), r \geq 0$, and $|a|,|\grave{a}| \leq a_{\mathrm{per}}$. Then

$$
\left\|\phi_{\mu, 1}^{a}-\phi_{\mu, 1}^{\grave{a}}\right\|_{W^{r, \infty}} \leq C(r) \mu^{1-r / 2}|a-\grave{a}| .
$$

(iii) Let $r \geq 0$ and $|a| \leq a_{\text {per. Then }}$

$$
\sup _{\mu \in\left(0, \mu_{\mathrm{per}}\right)}\left\|\psi_{\mu, 1}^{a}\right\|_{W^{r, \infty}} \leq C(r)|a| .
$$

Throughout the following estimates on the $h_{k}^{\mu}$ and $\ell_{k}^{\mu}$ terms, we recall that $a, \grave{a}, \mu \in(0,1)$, which will allow us to bound some terms quickly. Also, since $\bar{q}_{\star}<q_{\star}$, we have

$$
\|\boldsymbol{\eta}\|_{r, \bar{q}_{\star}} \leq\|\boldsymbol{\eta}\|_{r, q_{\star}}
$$

\section{D.3.1 Estimates on $h_{1}^{\mu}$}

We directly estimate

$$
\left\|h_{1}^{\mu}(\boldsymbol{\eta}, a)\right\|_{r, q_{\star}} \leq C(r) \mu\|\boldsymbol{\eta}\|_{r, q_{\star}} \quad \text { and } \quad\left\|h_{1}^{\mu}(\boldsymbol{\eta}, a)-h_{1}^{\mu}(\grave{\eta}, \grave{a})\right\|_{3, \bar{q}_{\star}} \leq C \mu\|\boldsymbol{\eta}-\grave{\boldsymbol{\eta}}\|_{3, \bar{q}_{\star}} .
$$

\section{D.3.2 Estimates on $h_{2}^{\mu}$}

We use the product estimate (D.22), the scaling estimate (D.24), and the periodic estimate (D.27) in Corollary 9 to bound

$$
\left\|h_{2}^{\mu}(\eta, a)\right\|_{r, q_{\star}} \leq C(r) \mu^{1-r / 2}|a| .
$$

Next, keeping $r$ arbitrary for later convenience, we estimate

$$
\left\|h_{2}^{\mu}(\boldsymbol{\eta}, a)-h_{2}^{\mu}(\grave{\eta}, \grave{a})\right\|_{r, \bar{q}_{\star}} \leq \underbrace{C|a-\grave{a}|\left\|\zeta_{c, 1}^{\mu} \phi_{\mu, 1}^{a}\right\|_{r, \bar{q}_{\star}}}_{I}+\underbrace{C|a|\left\|\zeta_{c, 1}^{\mu}\left(\phi_{\mu, 1}^{a}-\phi_{\mu, 1}^{\grave{a}}\right)\right\|_{r, \bar{q}_{\star}}}_{I I} .
$$

The same estimates that implied (D.30) also give

$$
I \leq C \mu^{1-r / 2}|a-\grave{a}| .
$$

For $I I$, since $\bar{q}_{\star}<q_{c}$, we use the massive decay borrowing estimate (D.26) and the various periodic estimates in (3.10) and Corollary 9 to bound

$$
I I \leq C \mu^{1-r / 2}|\grave{a}||a-\grave{a}| .
$$




\section{D.3.3 Estimates on $h_{3}^{\mu}$}

We use the product estimate (D.22) and the periodic estimate (D.27) to bound

$$
\left\|h_{3}^{\mu}(\eta, a)\right\|_{r, q_{\star}} \leq C(r) \mu^{1-r / 2}|a|\|\eta\|_{r, q_{\star}} .
$$

Next, keeping $r$ arbitrary for later convenience, we have

$$
\begin{aligned}
\left\|h_{3}^{\mu}(\eta, a)-h_{3}^{\mu}(\grave{\eta}, \grave{a})\right\|_{r, \bar{q}_{\star}} \leq & \underbrace{C\left(r, q_{\star}\right)|a-\grave{a}|\left\|\phi_{\mu, 1}^{a} \eta_{1}\right\|_{r, \bar{q}_{\star}}}_{I}+\underbrace{|\grave{a}|\left\|\left(\phi_{\mu, 1}^{a}-\phi_{\mu, 1}^{\grave{a}}\right) \eta_{1}\right\|_{r, \bar{q}_{\star}}}_{I I} \\
& +\underbrace{|\grave{a}|\left\|\phi_{\mu, 1}^{\grave{a}}\left(\eta_{1}-\grave{\eta}_{1}\right)\right\|_{r, \bar{q}_{\star}}}_{I I I} .
\end{aligned}
$$

As in Appendix 1, we first use the decay borrowing estimate (D.25), since $\bar{q}_{\star}<q_{\star}$, and find

$$
I \leq C \mu^{1-r / 2}|a-\grave{a}|\|\eta\|_{r, q_{\star}} .
$$

Next, we estimate

$$
I I I \leq C \mu^{1-r / 2}|\grave{a}|\|\eta-\grave{\eta}\|_{r, \bar{q}_{\star}}
$$

just as we did with (D.32). For $I I$, we use decay borrowing and the periodic estimates as we did to establish (D.31), and we find

$$
I I \leq C \mu^{1-r / 2}|\grave{a}|\|\eta\|_{r, q_{\star}}|a-\grave{a}| .
$$

\section{D.3.4 Estimates on $h_{4}^{\mu}$}

Since $h_{4}^{\mu}$ is quadratic in $\eta_{1}$, we have

$$
\left\|h_{4}^{\mu}(\eta, a)\right\|_{r, q_{\star}} \leq C(r)\|\eta\|_{r, q_{\star}}^{2}
$$

and

$$
\left\|h_{4}^{\mu}(\boldsymbol{\eta}, a)-h_{4}^{\mu}(\grave{\boldsymbol{\eta}}, \grave{a})\right\|_{3, \bar{q}_{\star}} \leq C\|\boldsymbol{\eta}+\grave{\boldsymbol{\eta}}\|_{r, \bar{q}_{\star}}\|\boldsymbol{\eta}-\grave{\boldsymbol{\eta}}\|_{3, \bar{q}_{\star}} .
$$

\section{D.3.5 Estimates on $h_{5}^{\mu}$}

The requisite estimates on $h_{5}^{\mu}$ are $\mathscr{O}(1)$ multiples of those for the $\ell_{k}^{\mu}$ terms, so we just proceed to establish those below.

\section{D.3.6 Estimates on $\ell_{1}^{\mu}$}

Theorem 1 implies

$$
\left\|\ell_{1}^{\mu}(\eta, a)\right\|_{r, q_{\star}} \leq C\left(r, q_{\star}\right) \mu^{2},
$$

and since $\ell_{1}^{\mu}$ is constant in both $\eta$ and $a$, its Lipschitz estimate is trivial. 


\section{D.3.7 Estimates on $\ell_{2}^{\mu}$}

We directly estimate

$$
\left\|\ell_{2}^{\mu}(\boldsymbol{\eta}, a)\right\|_{r, q_{\star}} \leq C(r) \mu\left(\|\boldsymbol{\eta}\|_{r, q_{\star}}+\|\boldsymbol{\eta}\|_{r, q_{\star}}^{2}\right)
$$

and

$$
\left\|\ell_{2}^{\mu}(\boldsymbol{\eta}, a)\right\|_{3, \bar{q}_{\star}} \leq C \mu\left(\|\boldsymbol{\eta}-\grave{\eta}\|_{3, \bar{q}_{\star}}+\|\boldsymbol{\eta}+\grave{\boldsymbol{\eta}}\|_{3, \bar{q}_{\star}}\|\boldsymbol{\eta}-\grave{\boldsymbol{\eta}}\|_{3, \bar{q}_{\star}}\right) .
$$

\section{D.3.8 Estimates on $\ell_{3}^{\mu}$}

These estimates are the same as those on $h_{3}^{\mu}$ from Appendix 1, except now we gain an extra factor of $\mu$ :

$$
\left\|\ell_{3}^{\mu}(\eta, a)\right\|_{r, q_{\star}} \leq C(r) \mu^{2-r / 2}|a|\|\eta\|_{r, q_{\star}}
$$

and (taking $r=3$ in the $h_{2}^{\mu}$ Lipschitz estimates)

$$
\left\|\ell_{3}^{\mu}(\boldsymbol{\eta}, a)-\ell_{3}^{\mu}(\grave{\boldsymbol{\eta}}, \grave{a})\right\|_{3, \bar{q}_{\star}} \leq C \mu^{2-r / 2}\left(|a-\grave{a}|\|\boldsymbol{\eta}\|_{3, \bar{q}_{\star}}+|\grave{a}|\|\boldsymbol{\eta}-\grave{\eta}\|_{3, \bar{q}_{\star}}+|\grave{a}||a-\grave{a}|\|\boldsymbol{\eta}\|_{3, \bar{q}_{\star}}\right) .
$$

\section{D.3.9 Estimates on $\ell_{4}^{\mu}$}

We recall from Theorem 2 that

$$
\phi_{\mu, 1}^{a}-\phi_{\mu, 1}^{0}=\mu v_{\mu}\left(\cos \left(\omega_{\mu}^{a} \cdot\right)-\cos \left(\omega_{\mu}^{0} \cdot\right)\right)+\mu \psi_{\mu, 1}^{a}\left(\omega_{\mu}^{a} \cdot\right),
$$

and so

$$
\ell_{4}^{\mu}(\boldsymbol{\eta}, a)=\underbrace{2 a \mu^{2} v_{\mu} \delta\left[\sigma_{c}\left(\cos \left(\omega_{\mu}^{a} \cdot\right)-\cos \left(\omega_{\mu}^{0} \cdot\right)\right)\right]}_{\ell_{41}^{\mu}(\boldsymbol{\eta}, a)}+\underbrace{2 a \mu^{2} \delta\left[\sigma_{c} \psi_{\mu, 1}^{a}\left(\omega_{\mu}^{a} \cdot\right)\right]}_{\ell_{42}^{\mu}(\boldsymbol{\eta}, a)} .
$$

Since $v_{\mu}=\mathscr{O}(1)$ and $q_{\star}<q_{c}$, we may use the decay borrowing estimate (D.25) and the periodic Lipschitz estimate (3.10) to bound

$$
\left\|\ell_{41}^{\mu}(\eta, a)\right\|_{r, q_{\star}} \leq C(r) \mu^{2} a^{2} .
$$

Next, the scaling estimate (D.24) and the periodic estimates (D.27) and (D.29) imply

$$
\left\|\ell_{42}^{\mu}(\eta, a)\right\|_{r, q_{\star}} \leq C\left(r, q_{\star}\right) \mu^{2}|a|\left\|\psi_{\mu, 1}^{a}\left(\omega_{\mu}^{a} \cdot\right)\right\|_{W^{r, \infty}} \leq C\left(r, q_{\star}\right) \mu^{2-r / 2} a^{2} .
$$

All together, we conclude

$$
\left\|\ell_{4}^{\mu}(\eta, a)\right\|_{r, q_{\star}} \leq C(r) \mu^{2-r / 2} a^{2} .
$$

For the Lipschitz estimates, we first calculate

$$
\begin{aligned}
\ell_{41}^{\mu}(\boldsymbol{\eta}, a)-\ell_{41}^{\mu}(\grave{\eta}, \grave{a})= & \underbrace{2 \mu^{2} v_{\mu}(a-\grave{a}) \delta\left[\sigma_{c}\left(\cos \left(\omega_{\mu}^{a} \cdot\right)-\cos \left(\omega_{\mu}^{0} \cdot\right)\right)\right]}_{I} \\
& +\underbrace{2 \mu^{2} v_{\mu}|\grave{a}| \delta\left[\sigma_{c}\left(\cos \left(\omega_{\mu}^{a} \cdot\right)-\cos \left(\omega_{\mu}^{\grave{a}} \cdot\right)\right)\right]}_{I I} .
\end{aligned}
$$


Then to bound $I$ and $I I$, we use the decay borrowing estimate (D.25) and the periodic Lipschitz estimate (3.10) and find

$$
\|I\|_{3, q} \leq C \mu^{2}|a||a-\grave{a}| \quad \text { and } \quad\|I I\|_{3, q} \leq C \mu^{2}|\grave{a} \| a-\grave{a}| .
$$

\section{D.3.10 Estimates on $\ell_{5}^{\mu}$}

These estimates are effectively the same as those on $h_{2}^{\mu}$ from Appendix 1, except now we gain an extra factor of $\mu^{2}$ :

$$
\left\|\ell_{5}^{\mu}(\eta, a)\right\|_{r, q_{\star}} \leq C(r) \mu^{3-r / 2}|a|
$$

and (taking $r=3$ in the $h_{2}^{\mu}$ Lipschitz estimates)

$\left\|\ell_{5}^{\mu}(\boldsymbol{\eta}, a)-\ell_{5}^{\mu}(\grave{\boldsymbol{\eta}}, \grave{a})\right\|_{3, \bar{q}_{\star}} \leq C\left(\mu^{3 / 2}|a-\grave{a}|+\mu^{2}|\grave{a}|\|\boldsymbol{\eta}-\grave{\boldsymbol{\eta}}\|_{3, \bar{q}_{\star}}+\mu^{3 / 2}|\grave{a}|\|\boldsymbol{\eta}\|_{r, \bar{q}_{\star}}|a-\grave{a}|\right)$.

\section{D.3.11 Estimates on $\ell_{6}^{\mu}$}

First, we use the decay borrowing estimate (D.25) to find

$$
\begin{aligned}
& \left\|\ell_{6}^{\mu}(\eta, a)\right\|_{r, q_{\star}} \leq C\left(r, q_{\star}\right) \mu^{2}|a|\left\|\sigma_{c}\left(\cos \left(\omega_{\mu} \cdot\right)-\cos \left(\Omega_{\mu} \cdot\right)\right)\right\|_{r, q_{\star}} \\
& \quad \leq C\left(r, q_{\star}\right) \mu^{2}|a|\left\|\sigma_{c}\right\|_{r, q_{\star}}\left|\omega_{\mu}-\Omega_{\mu}\right| .
\end{aligned}
$$

Next, recall from part (iii) of Lemma 2 that $\omega_{\mu}-\Omega_{\mu}=\mathscr{O}\left(\mu^{3 / 2}\right)$, and so we conclude

$$
\left\|\ell_{6}^{\mu}(\eta, a)\right\|_{r, q_{\star}} \leq C\left(r, q_{\star}\right) \mu^{7 / 2}|a| .
$$

The same ideas give the Lipschitz estimate

$$
\left\|\ell_{6}^{\mu}(\eta, a)-\ell_{6}^{\mu}(\grave{\eta}, \grave{a})\right\|_{3, \bar{q}_{\star}} \leq C \mu^{7 / 2}|a-\grave{a}| .
$$

\section{References}

1. Amick, C.J., Toland, J.F.: Solitary waves with surface tension I. Trajectories homoclinic to periodic orbits in four dimensions. Arch. Ration. Mech. Anal. 118(1), 37-69 (1992)

2. Beale, J.T.: Water waves generated by a pressure disturbance on a steady stream. Duke Math. J. 47(2), 297-323 (1980)

3. Beale, J.T.: Exact solitary water waves with capillary ripples at infinity. Commun. Pure Appl. Math. 44(2), 211-257 (1991)

4. Bonanomi, L., Theocharis, G., Daraio, C.: Wave propagation in granular chains with local resonances. Phys. Rev E Stat. Nonlinear Soft Matter Phys. 91, 033208 (2015)

5. Boyd, J.P.: Weakly Nonlocal Solitary Waves and Beyond-All-Orders Asymptotics, Mathematics and Its Applications, vol. 442. Kluwer Academic Publishers, Dordrecht (1998)

6. Chong, C., Porter, M.A., Kevrekidis, P.G., Daraio, C.: Nonlinear coherent structures in granular crystals. J. Phys. Condens. Matter 29(41), 413003 (2016)

7. Crandall, M.G., Rabinowitz, P.H.: Bifurcation from simple eigenvalues. J. Funct. Anal. 8, 321-340 (1971)

8. Dauxois, T.: Fermi, Pasta, Ulam, and a mysterious lady. Phys. Today 61(1), 55-57 (2008)

9. English, J.M., Pego, R.L.: On the solitary wave pulse in a chain of beads. Proc. Am. Math. Soc. 133(6), 1763-1768 (2005)

10. Faver, T.E.: Nanopteron-stegoton traveling waves in mass and spring dimer Fermi-Pasta-Ulam-Tsingou lattices. Ph.D. thesis, Drexel University, Philadelphia, PA (2018)

11. Faver, T.E.: Nanopteron-stegoton traveling waves in spring dimer Fermi-Pasta-Ulam-Tsingou lattices. Q. Appl. Math. 78, 363-429 (2020)

12. Faver, T.E., Goodman, R.H., Wright, J.D.: Solitary waves in mass-in-mass lattices for antiresonance mass ratios. arXiv preprint arXiv:2002.05573 
13. Faver, T.E., Hupkes, H.J.: Micropteron traveling waves in diatomic Fermi-Pasta-Ulam-Tsingou lattices under the equal mass limit. Phys. D Nonlinear Phenom. 410 (2020)

14. Faver, T.E., Wright, J.D.: Exact diatomic Fermi-Pasta-Ulam-Tsingou solitary waves with optical band ripples at infinity. SIAM J. Math. Anal. 50(1), 182-250 (2018)

15. Fermi, E., Pasta, J., Ulam, S.: Studies of nonlinear problems. Lect. Appl. Math. 12, 143-56 (1955)

16. Friesecke, G., Pego, R.L.: Solitary waves on FPU lattices. I. Qualitative properties, renormalization and continuum limit. Nonlinearity 12(6), 1601-1627 (1999)

17. Friesecke, G., Wattis, J.A.D.: Existence theorem for solitary waves on lattices. Commun. Math. Phys. 161(2), 391-418 (1994)

18. Kevrekidis, P.G., Vainchtein, A., Serra-Garcia, M., Daraio, C.: Interaction of traveling waves with masswith-mass defects within a Hertzian chain. Phys. Rev. E Stat. Nonlinear Soft Matter Phys. 87, 042911 (2013)

19. Gaison, J., Moskow, S., Wright, J.D., Zhang, Q.: Approximation of polyatomic FPU lattices by KdV equations. Multiscale Model. Simul. 12(3), 953-995 (2014)

20. Gantzounis, G., Serra-Garcia, M., Homma, K., Mendoza, J.M., Daraio, C.: Granular metamaterials for vibration mitigation. J. Appl. Phys. 114, 093514 (2013)

21. Giardetti, N., Shapiro, A., Windle, S., Wright, J.D.: Metastability of solitary waves in diatomic FPUT lattices. Math. Eng. 1(3), 419-433 (2019)

22. Herrmann, M., Matthies, K.: Asymptotic formulas for solitary waves in the high-energy limit of FPU-type chains. Nonlinearity 28, 2767-2789 (2015)

23. Herrmann, M., Matthies, K.: Uniqueness of solitary waves in the high-energy limit of FPU-type chains. In: Gurevich, P., Hell, J., Sandstede, B., Scheel, A. (eds.) Patterns of Dynamics, Springer Proceedings in Mathematics \& Statistics, vol. 205, pp. 3-15. Springer, Berlin (2017)

24. Herrmann, M., Matthies, K.: Stability of high-energy solitary waves in Fermi-Pasta-Ulam-Tsingou chains. Trans. Am. Math. Soc. 372, 3425-3486 (2019)

25. Hoffman, A., Wright, J.D.: Nanopteron solutions of diatomic Fermi-Pasta-Ulam-Tsingou lattices with small mass-ratio. Phys. D 358, 33-59 (2017)

26. Iooss, G.: Travelling waves in the Fermi-Pasta-Ulam lattice. Nonlinearity 13(3), 849-866 (2000)

27. Iooss, G., Kirchgässner, K.: Travelling waves in a chain of coupled nonlinear oscillators. Commun. Math. Phys. 211(2), 439-464 (2000)

28. Ji, J.Y., Hong, J.: Existence criterion of solitary waves in a chain of grains. Phys. Lett. A 260, 60-61 (1999)

29. Johnson, M.A., Wright, J.D.: Generalized solitary waves in the gravity-capillary Whitham equation. Stud. Appl. Math. 144, 102-130 (2020)

30. Kevrekidis, P.G.: Non-linear waves in lattices: past, present, future. IMA J. Appl. Math. 76(3), 389-423 (2011)

31. Kevrekidis, P.G., Stefanov, A.G., Xu, H.: Traveling waves for the mass in mass model of granular chains. Lett. Math. Phys. 106(8), 1067-1088 (2016)

32. Kim, E., Li, F., Chong, C., Theocharis, G., Yang, J., Kevrekidis, P.G.: Highly nonlinear wave propagation in elastic woodpile periodic structures. Phys. Rev. Lett. 114(11), 118002 (2015)

33. Li, F., Anzel, P., Yang, J., Kevrekidis, P.G., Daraio, C.: Granular acoustic switches and logic elements. Nat. Commun. 5, 5311 (2014)

34. Lombardi, E.: Oscillatory Integrals and Phenomena Beyond all Algebraic Orders with Applications to Homoclinic Orbits in Reversible Systems. Lecture Notes in Mathematics, vol. 1741. Springer, Berlin (2000)

35. Lustri, C., Porter, M.A.: Nanoptera in a period-2 Toda chain. SIAM J. Appl. Dyn. Syst. 17(2), 1182-1212 (2018)

36. Lustri, C.J.: Nanoptera and Stokes curves in the 2-periodic Fermi-Pasta-Ulam-Tsingou equation. ArXiv preprint arXiv:1905.07092

37. MacKay, R.S.: Solitary waves in a chain of beads under Hertz contact. Phys. Lett. A 251(3), 191-192 (1999)

38. Nesterenko, V.F.: Propagation of nonlinear compression pulses in granular media. J. Appl. Mech. Tech. Phys. 24(5), 733-743 (1983)

39. Nesterenko, V.F.: Dynamics of Heterogeneous Materials. Shock Wave and High Pressure Phenomena. Springer, Berlin (2001)

40. Ni, X., Rizzo, P., Yang, J., Katri, D., Daraio, C.: Monitoring the hydration of cement using highly nonlinear solitary waves. NDT E Int. 52, 76-85 (2012)

41. Pankov, A.: Travelling Waves and Periodic Oscillations in Fermi-Pasta-Ulam Lattices. Imperial College Press, Singapore (2005) 
42. Spadoni, A., Daraio, C.: Generation and control of sound bullets with a nonlinear acoustic lens. Proc. Natl. Acad. Sci. 107(16), 7230-7234 (2010)

43. Starosvetsky, Y., Vainchtein, A.: Solitary waves in FPU lattices with alternating bond potentials. Mech. Res. Commun. 93, 148-153 (2018)

44. Stefanov, A., Kevrekidis, P.: On the existence of solitary traveling waves for generalized Hertzian chains. J. Nonlinear Sci. 22, 327-349 (2012)

45. Stefanov, A., Kevrekidis, P.: Traveling waves for monomer chains with precompression. Nonlinearity 26, 539-564 (2013)

46. Sun, S.M., Shen, M.C.: Exponentially small estimate for the amplitude of capillary ripples of a generalized solitary wave. J. Math. Anal. Appl. 172(2), 533-566 (1993)

47. Toda, M.: Theory of Nonlinear Lattices, Springer Series in Solid State Sciences, vol. 20. Springer, Berlin (1978)

48. Vainchtein, A., Starosvetsky, Y., Wright, J.D., Perline, R.: Solitary waves in diatomic chains. Phys. Rev. E 93, 042210 (2016)

49. Venney, C.R., Zimmer, J.: Travelling lattice waves in a toy model of Lennard-Jones interaction. Q. Appl. Math. 72(1), 65-84 (2014)

50. Vorotnikov, K., Starosvetsky, Y., Theocharis, G., Kevrekidis, P.G.: Wave propagation in a strongly nonlinear locally resonant granular crystal. Phys. D 365, 27-41 (2018)

51. Xu, H., Kevrekidis, P.G., Stefanov, A.: Traveling waves and their tails in locally resonant granular systems. J. Phys. A Math. Theor. 48, 195204 (2015)

52. Yang, J., Sangiorgio, S.N., Borkowski, S.L., Silvestro, C., De Nardo, L., Daraio, C., Ebramzadeh, E.: Sitespecific quantification of bone quality using highly nonlinear solitary waves. J. Biomech. Eng. 134(10), $101001(2012)$

53. Zeidler, E.: Applied Functional Analysis, Applied Mathematical Sciences, vol. 109. Springer, New York (1995). Main principles and their applications

Publisher's Note Springer Nature remains neutral with regard to jurisdictional claims in published maps and institutional affiliations. 